J. Math. Biol. (1994) 33:163-192

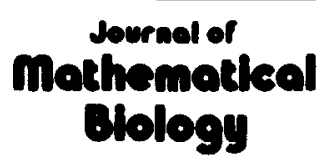

(C) Springer-Verlag 1994

\title{
Existence and uniqueness of a sharp travelling wave in degenerate non-linear diffusion Fisher-KPP equations
}

\author{
Faustino Sánchez-Garduño ${ }^{1,2}$, Philip K. Maini ${ }^{1}$ \\ ${ }^{1}$ Centre for Mathematical Biology, Mathematical Institute, University of Oxford, \\ 24-29 St Giles', Oxford OX1 3LB, UK \\ ${ }^{2}$ Departamento de Matemáticas. Facultad de Ciencias. UNAM. Circuito Exterior, C.U., \\ México, 04510 D.F. Mexico
}

Received 2 November 1993; received in revised version 2 February 1994

\begin{abstract}
In this paper we use a dynamical systems approach to prove the existence of a unique critical value $c^{*}$ of the speed $c$ for which the degenerate density-dependent diffusion equation $u_{t}=\left[D(u) u_{x}\right]_{x}+g(u)$ has: 1 . no travelling wave solutions for $0<c<c^{*}$, 2. a travelling wave solution $u(x, t)=$ $\phi\left(x-c^{*} t\right)$ of sharp type satisfying $\phi(-\infty)=1, \quad \phi(\tau)=0 \quad \forall \tau \geqq \tau^{*}$; $\phi^{\prime}\left(\tau^{*-}\right)=-c^{*} / D^{\prime}(0), \phi^{\prime}\left(\tau^{*+}\right)=0$ and 3 . a continuum of travelling wave solutions of monotone decreasing front type for each $c>c^{*}$. These fronts satisfy the boundary conditions $\phi(-\infty)=1, \phi^{\prime}(-\infty)=\phi(+\infty)=\phi^{\prime}(+\infty)=0$. We illustrate our analytical results with some numerical solutions.
\end{abstract}

Key words: Travelling waves - Non-linear diffusion equations - Sharp solutions - Wavespeed - Degenerate diffusion

\section{Introduction}

In spite of its biological importance, the introduction of dispersal into mathematical models to describe the space distribution patterns of species is relatively new. Continuous models to describe the spread of biological populations in their habitat can be derived in two ways: 1 . Random walks followed by the so-called diffusion approximation and 2. Continuous media dynamics, based on conservation laws.

The first systematic model to describe the space distribution of individuals of a single population was derived by Skellam [30]. He used a random walk approach to deduce the equation $u_{t}=D \nabla^{2} u+g(u)$ where $u=u(r, t)$ is the population density at position $r$ and time $t, D$ is a positive constant and $g$ is the growth rate. In his approach the basic assumption was that the habitat is homogeneous. From the modelling viewpoint this means that the probability $p$ that an individual of the population moves from one point $\boldsymbol{r}_{1}$ to another 
point $\boldsymbol{r}_{2}$ in a small time interval $\tau$ is the same as that of moving from $\boldsymbol{r}_{2}$ to $\boldsymbol{r}_{1}$ in the same time interval $\tau$, i.e., the probability is a space-symmetric function. In mathematical terms we have $p\left(\boldsymbol{r}_{1}, \boldsymbol{r}_{2}\right)=p\left(\boldsymbol{r}_{2}, \boldsymbol{r}_{1}\right)$ for all pairs of points $\boldsymbol{r}_{1}$ and $\boldsymbol{r}_{2}$.

However, as pointed out by Skellam [31] himself, there are biological (mating, attracting and repelling substances, overcrowding, spatial distribution of food, social behaviour, etc.) and physical (light, temperature, humidity, etc.) factors which influence population dispersal. The introduction of some of these factors into the derivation of the corresponding models implies that the probability $p$ is no longer a space-symmetric function. It could depend on local biological and physical conditions such as population density or concentration of a chemoattractant or chemorepellant, for instance.

In this paper we restrict ourselves to the case in which the probability is a density-dependent function. In Sect. 2 we review several density-dependent dispersal models and show that their flux terms are all special cases of a more general case. We then consider a special type of solution to this general problem, namely, travelling wave solutions ( t.w.s.). In Sect. 3 we briefly review existence results for t.w.s. for the linear and non-linear density-dependent diffusion Fisher-KPP models. We extend these results to the general nonlinear degenerate diffusion equation. Section 4 contains a review of the local non-linear analysis of the system of ordinary differential equations (ODEs) associated with the dynamics of the t.w.s. for the partial differential equation (PDE). In Sect. 5, by analysing the global phase portrait of the ODE system for sufficiently small and then for sufficiently large values of $c$, we obtain the non-existence of t.w.s. and a result which assures the existence of a continuum of t.w.s. of front type, respectively. Section 6 contains the result which ensures the existence of a saddle-saddle heteroclinic trajectory i.e., the existence of a travelling wave solution of sharp type. In Sect. 7 we prove the uniqueness of the sharp front. The analytical results are illustrated by considering a few examples in Sect. 8.

\section{Density-dependent models}

Density-dependent dispersal has been observed in many biological populations. By way of example we briefly mention a few of them. Myers and Krebs [23] studied the population density cycles in small rodents, in particular the highest incidence of dispersal during periods of population growth and the effect of the stress produced by high densities in the population. Carl [7] observed that Arctic ground squirrels migrate from crowded areas into sparsely populated areas. Morisita (in [29]) studied the spatial segregation of the ant-lion Glenuroides japonicus.

Several models have been proposed to describe the density-dependent dispersal phenomenon. The first density-dependent diffusion models in an ecological context were constructed by Gurney and Nisbet $[14,15]$. By using 
a random walk approach they deduced the equation

$$
\frac{\partial u}{\partial t}=G(\boldsymbol{r}) u-\nabla \cdot J(\boldsymbol{r}, t)
$$

for a single species of density $u$ where $G$ is the intrinsic rate of growth of the population and the flux $J$ takes different forms depending on the type of movement involved. For biased motion $J=-D \nabla u-\lambda u \nabla u$ and for directed motion $\boldsymbol{J}=-k u \nabla u$.

In the remainder of this section we consider only purely diffusive phenomena, that is, we concentrate on the flux term in (1).

Gurtin and MacCamy [16], by using a continuum mechanics approach and assuming that the average velocity $v$ of the population is an isotropic function of $u$ and $\nabla u$ i.e., that for any orthogonal transformation $Q, Q v(u, \nabla u)=v(u, Q \nabla u)$, derived the equation

$$
\frac{\partial u}{\partial t}=\phi^{\prime}(u) \nabla^{2} u+\phi^{\prime \prime}(u)\|\nabla u\|^{2}
$$

where $\phi(0)=\phi^{\prime}(0)$ and $\phi^{\prime}(u)>0 \forall u>0$.

Montroll and West [21] derived a one-dimensional model for a single species by using a random walk approach. Their basic assumption was that each step of length $a$ of an individual is determined by the population density at the nearest (left and right) points from the departure point. Their model is

$$
\frac{\partial u}{\partial t}=[D-2 \psi w(u) u] \frac{\partial^{2} u}{\partial x^{2}}-2 \psi\left[\frac{\partial u}{\partial x}\right]^{2} \frac{d}{d u}[w(u) u],
$$

where $D=a^{2} / 2 \tau, \psi=\mu a^{2} \tau$ and $w$ is a weighting function whose value tells us the direction of motion. For example, if $w$ is constant, then the bias depends on the gradient of the population.

Based on the results of Morisita's experiments on the ant-lion Glenuroides japonicus, Shiguesada et al. [29] developed a mathematical model to analyse the spatial distribution pattern and the effect of non-linear diffusion on the spatial segregation of a species. By analysing the flux of ants throughout a compartmentally divided habitat, they obtained the equation

$$
\frac{\partial u}{\partial t}=(\alpha+2 \beta u) \frac{\partial^{2} u}{\partial x^{2}}+2 \beta\left[\frac{\partial u}{\partial x}\right]^{2}+\frac{d}{d x}\left[u U^{\prime}(x)\right]
$$

where $U(x)$ is a space-dependent function which measures the favourableness potential of the habitat at the point $x$.

Aronson [4] denoted by $u_{l}^{n}$ and $p_{j}\left(u_{l}\right)$, respectively, the population density at locus al at time $n \tau$ and the probability of a jump of $a j$ units starting from locus al given that the population density at al is $u_{l}$. By assuming that $p_{j}(\cdot)=0$ for sufficiently large $|j|$ and that the conservation law $\sum_{j \in Z} p_{j}(u)=1$ holds, he proved the following recursion relationship

$$
u_{l}^{n+1}=\sum_{j \in Z} u_{l-j}^{n} p_{j}\left(u_{l-j}^{n}\right), \quad j \in Z, n \in Z^{+} .
$$


Furthermore, he then showed that $u$ satisfies

$$
\frac{\partial u}{\partial t}=\frac{\lambda}{2} \frac{\partial^{2}}{\partial x^{2}}[u \phi(u)]-\lambda \frac{\partial}{\partial x}[u \psi(u)],
$$

where

$$
\sum_{j \in Z} j^{2} p_{j}\left(u_{l}^{n}\right) \rightarrow \phi(u) \text { and } \frac{1}{a} \sum_{j \in Z} j p_{j}\left(u_{l}^{n}\right) \rightarrow \psi(u),
$$

$n \tau \rightarrow t, a l \rightarrow x$ and $a^{2} / \tau \rightarrow \lambda$ as $\tau, a \rightarrow 0$ and $l, m \rightarrow \infty$. The second term in the right hand side of (5) can be interpreted as a drift term which vanishes if $p_{j}(\cdot)=p_{-j}(\cdot)$.

If, instead of supposing that the probabilities depend on the local population densities, it is assumed that they depend on the average between the beginning and the end of the step, it can be shown [4] that

$$
\frac{\partial u}{\partial t}=\frac{\partial}{\partial x}\left[p(u) \frac{\partial u}{\partial x}\right] \text {. }
$$

Note that if we define $D$ such that $\phi(u)=\int_{0}^{u} D(s) d s$ then model (2) takes the form

$$
\frac{\partial u}{\partial t}=D(u) \nabla^{2} u+D^{\prime}(u)\|\nabla u\|^{2}
$$

with $D(0)=0$ and $D(u)>0 \quad \forall u$. Observe also that if we set $\mathscr{D}(u)=[D-2 \psi w(u) u]$ then (3) can be written as

$$
\frac{\partial u}{\partial t}=\mathscr{D}(u) \frac{\partial^{2} u}{\partial x^{2}}+\mathscr{D}^{\prime}(u)\left[\frac{\partial u}{\partial x}\right]^{2} .
$$

Also, if we set $\mathscr{D}(u)=\phi(u)+u \phi^{\prime}(u)$ with zero drift term in (5), then this equation takes the form (8). Furthermore, setting $D(u)=(\alpha+2 \beta u)$ and imposing a constant potential environment $U$ in model (4), leads to the equation

$$
\frac{\partial u}{\partial t}=D(u) \frac{\partial^{2} u}{\partial x^{2}}+D^{\prime}(u)\left[\frac{\partial u}{\partial x}\right]^{2}
$$

Hence, models (2), (3) and (4) are all special cases of the one-dimensional version of (7). Therefore, we propose to analyse travelling wave solutions for a reaction-diffusion model in which the diffusive term takes this general form.

\section{Brief review of existence of t.w.s. for Fisher-KPP equations}

In this paper we study the problem of existence of t.w.s., $u(x, t)=$ $\phi(x-c t) \equiv \phi(\xi)$ for the parabolic equation

$$
\frac{\partial u}{\partial t}=\frac{\partial}{\partial x}\left[D(u) \frac{\partial u}{\partial x}\right]+g(u)
$$


where the functions $D$ and $g$ are defined on the interval $[0,1]$ and satisfy the following conditions:

1. $g(0)=g(1)=0, g(u)>0 \forall u \in(0,1)$,

2. $g \in C_{[0,1]}^{2}$ with $g^{\prime}(0)>0$ and $g^{\prime}(1)<0$,

3. $D(0)=0$ with $D(u)>0 \forall u \in(0,1]$,

4. $D \in C_{[0,1]}^{2}$ with $D^{\prime}(u)>0$ and $D^{\prime \prime}(u) \neq 0 \forall u \in[0,1]$,

with initial condition $u(x, 0)=u_{0}(x)$, where $u_{0}$ is any piecewise differentiable function with $0 \leqq u_{0}(x) \leqq 1$ and boundary conditions

$$
\lim _{\xi \rightarrow-\infty} \phi(\xi)=1 \text { and } \lim _{\xi \rightarrow+\infty} \phi(\xi)=0 .
$$

We also require that $0 \leqq \phi(\xi) \leqq 1$ for all $\xi \in \boldsymbol{R}$.

The ecological meaning of t.w.s. for (10) is that the individuals of the population disperse throughout the habitat in a constant density profile moving with a constant speed.

Because of condition 3, (10) degenerates into an ODE at $u=0$, while for $u>0(10)$ is of parabolic type. This property leads to existence of a particular kind of travelling wave solution which can be defined as follows.

Definition 1. If there exists a value $c^{*}>0$ of the speed $c$, and a value $\xi^{*} \in(-\infty,+\infty)$ of $\xi$ such that $u(x, t)=\phi\left(x-c^{*} t\right)=\phi(\xi)$ satisfies:

1. $D(\phi) \phi^{\prime \prime}+c^{*} \phi^{\prime}+D^{\prime}(\phi)\left[\phi^{\prime}\right]^{2}+g(\phi)=0, \forall \xi \in\left(-\infty, \xi^{*}\right)$,

2. $\phi(-\infty)=1, \phi\left(\xi^{*-}\right)=\phi\left(\xi^{*+}\right)=0$ and $\phi(\xi)=0 \forall \xi \in\left(\xi^{*},+\infty\right]$,

3. $\phi^{\prime}\left(\xi^{*-}\right)=-\frac{c^{*}}{D^{\prime}(0)}, \phi^{\prime}\left(\xi^{*+}\right)=0$, and $\phi^{\prime}(\xi)<0 \forall \xi \in\left(-\infty, \xi^{*}\right)$,

then the function $u(x, t)=\phi\left(x-c^{*} t\right)$ is called a travelling wave solution of sharp type for (10).

Before embarking on the analysis of this degenerate problem we briefly review the results on existence of t.w.s. for the standard Fisher-KPP equation. The first systematic analysis on the existence of t.w.s. for the constant-diffusion equation $u_{t}=D u_{x x}+g(u)$ where $D$ is a positive constant, $g(0)=g(1)=0$, $g(u)>0 \forall u \in(0,1), g^{\prime}(0)>0$ and $g^{\prime}(1)<0$ appeared in two separate works due to Fisher [12] and Kolmogorov et al. [19]. In both cases the biological motivation was the analysis of the spread of an advantageous gene in a population living in a one-dimensional habitat. In fact the main ideas of the methodology introduced by Kolmogorov et al. are still used today. A modern version of their result on existence of t.w.s. can be seen in Fife [11] and Britton [6], and may be stated as follows: For each $c \geqq c^{*}$ the above equation possesses a monotonic decreasing front $u(x, t)=\phi(x-c t)$ satisfying the boundary conditions $\phi(-\infty)=1$ and $\phi(+\infty)=0$, if and only if $c^{*}$ is such that $2 \sqrt{g^{\prime}(0) D} \leqq c^{*} \leqq 2 \sqrt{v D}$ where $v=\sup g(u) / u$ and the sup is taken on $(0,1)$.

For the non-linear diffusion $u_{t}=\left[D(u) u_{x}\right]_{x}+g(u)$ where $D$ is a strictly positive function on $[0,1]$ and the kinetic part $g$ is as in the classic FisherKPP equation, Hadeler [17] gave the lower bound on $c$ for the existence of t.w.s. of front type. He also found ([18]) an important relationship between 
the ODE system associated with the t.w.s. of the Fisher-KPP equation and the corresponding ODE system for the t.w.s. of the more general equation $u_{t}=1 / m(u)\left[D(u) u_{x}\right]_{x}+g(u)$. Engler [10] proved that the above equation possesses t.w.s. if and only if the equation $u_{t}=u_{x x}+D(u) g(u)$ also has t.w.s.

Except for particular cases of the degenerate equation (10), the problem of existence of t.w.s. for this equation is still not completely solved. For the equation $u_{t}=\left[u u_{x}\right]_{x}+u(1-u)$ a complete analysis has been carried out by Aronson [4], Newman [24] and Murray [22]. The result may be stated as follows. There exists a value $c^{*}=1 / \sqrt{2}$ of $c$ such that: 1 . For $0<c<c^{*}$ there are no t.w.s., 2. For $c=c^{*}$ there is a travelling wave solution of sharp type (the explicit form is given in $[22,24]$ ); 3. For each $c>c^{*}$ there is a t.w.s. of front type satisfying the boundary conditions $\phi(-\infty)=1$ and $\phi(+\infty)=0$. Newman [25] studied the more general system $u_{t}=\left[u^{n} u_{x}\right]_{x}+u(1-u)$ using Lyapunov functionals. De Pablo and Vázquez [9] studied the equation $u_{t}=\left[u^{m}\right]_{x x}+\lambda u^{n}(1-u)$ with $m>1$ and $\lambda>0$ They proved the existence of a unique travelling wave of sharp type for $\lambda=1 / m$ and $(m+n) \geqq 0$.

\section{Review: the positivity of $c$ and the non-linear local analysis}

Let us rewrite (10) as

$$
\frac{\partial u}{\partial t}=D(u) \frac{\partial^{2} u}{\partial x^{2}}+D^{\prime}(u)\left[\frac{\partial u}{\partial x}\right]^{2}+g(u) .
$$

Note we note that if a travelling wave solution $u(x, t)=\phi(x-c t)$ of (11) exists, then it must satisfy the following second order ODE

$$
D(\phi(\xi)) \phi^{\prime \prime}+c \phi^{\prime}(\xi)+D^{\prime}(\phi(\xi))\left[\phi^{\prime}(\xi)\right]^{2}+g(\phi(\xi))=0 .
$$

Here dash means the derivative with respect to $\phi$ on $D$ and with respect to $\xi$ on $\phi$. Let $\xi^{*} \in(-\infty,+\infty]$ be such that $\phi(-\infty)=1$ and $\phi(\xi)=0 \forall \xi \in\left[\xi^{*},+\infty\right)$.

The following proposition holds:

Proposition 1. For suitable smoothness conditions on the functions $D$ and $g$ on the interval $[0,1]$, for $\xi^{*}$ as in Definition 1, and for the two types of behaviour of $\phi(\xi)$ in Fig 1 , we have

$$
c=\frac{\int_{0}^{1} D(w) g(w) d w}{\int_{-\infty}^{\xi^{*}} D(\phi(s))\left[\phi^{\prime}(s)\right]^{2} d s} \geqq 0 .
$$

Proof. See Sánchez-Garduño and Maini [27].

Our approach uses the result that for well-posed problems, to look for t.w.s. for a non-linear diffusion equation is equivalent to looking for the set of parameters (in which the speed $c$ is included) for which there exist heteroclinic trajectories of the system of ODEs which arises from transforming the original problem into travelling wave coordinates. (See Sánchez-Garduño [26]). Therefore the first part of the study of existence of t.w.s. for (10) consists of 


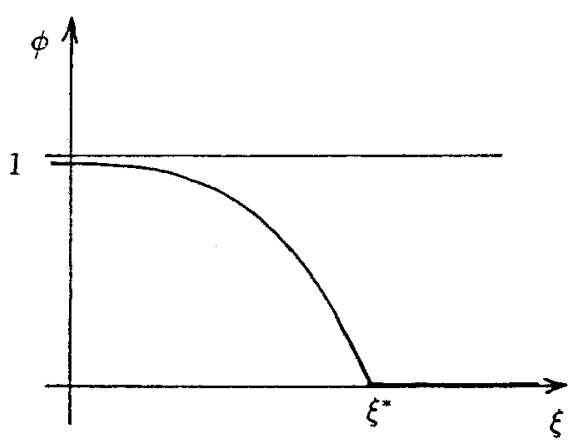

a

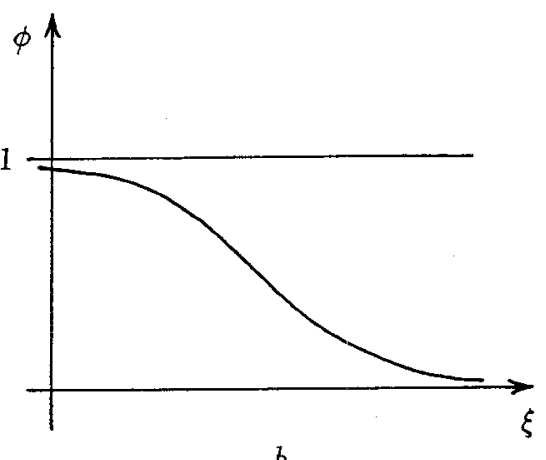

$b$

Fig. 1a, b. Different behaviours of t.w.s. to (10). a Monotone decreasing with discontinuous derivative in $\xi^{*}$. The left derivative tends to $\phi^{\prime}\left(\xi^{*-}\right) \neq 0$, the right derivative tends to $\phi^{\prime}\left(\xi^{*+}\right)=0$. This is the so-called sharp type front. b Monotone decreasing with continuous derivative. Here $\lim _{\xi \rightarrow+\infty} \phi(\xi)=\lim _{\xi \rightarrow+\infty} \phi^{\prime}(\xi)=0$

determining the local behaviour of the trajectories. Setting $v=\phi^{\prime}$ in (12) we have the following system of ODEs

$$
\begin{aligned}
\phi^{\prime} & =v \\
D(\phi) v^{\prime} & =-c v-D^{\prime}(\phi) v^{2}-g(\phi) .
\end{aligned}
$$

Since $D(0)=0$ this system possesses a singularity at $\phi=0$. We can remove it by introducing the parameter $\tau$ such that

$$
\frac{d \tau}{d \xi}=\frac{1}{D(\phi(\xi))} \Rightarrow \tau(\xi)=\int_{0}^{\xi} \frac{d s}{D(\phi(s))} .
$$

Except at $\phi=0$, where $\frac{d \tau}{d \xi}$ is not defined, $\frac{d \tau}{d \xi}>0$. Thus $\tau$ has an inverse $\tau^{-1}$ which in principle can be obtained from (15). Thus we have

and we obtain

$$
\phi(\xi)=\phi(\tau(\xi)) \quad \text { and } \quad v(\xi)=v(\tau(\xi))
$$

$$
\phi^{\prime}(\xi)=\frac{d \phi}{d \tau}(\tau) \frac{1}{D(\phi(\xi))} \quad \text { and } \quad v^{\prime}(\xi)=\frac{d v}{d \tau}(\tau) \frac{1}{D(\phi(\xi))}
$$

Substituting $\phi^{\prime}(\xi)$ and $v^{\prime}(\xi)$ into (14) we have the new system without the singularity

$$
\begin{aligned}
& \dot{\phi}=D(\phi) v \equiv F(\phi, v) \\
& \dot{v}=-c v-D^{\prime}(\phi) v^{2}-g(\phi) \equiv G(\phi, v)
\end{aligned}
$$

where the dot denotes differentation with respect to $\tau$.

Note that systems (14) and (18) are topologically equivalent in the positive half plane $\{(\phi, v) \mid \phi>0,-\infty<v<+\infty\}$. This is true because (16) defines a re-parametrization of the trajectories which, according to (17), preserves the orientation. In the remaining part of this section we only sketch the non-linear local analysis of the phase portrait of (18). Full details can be seen in [27]. 
Since $D^{\prime}(0)>0$, the system (18) has three equilibrium points: $P_{0}=(0,0)$, $P_{1}=(1,0)$ and $P_{c}=\left(0,-c / D^{\prime}(0)\right)$. The local behaviour of the trajectories of (18) can be obtained as usual by analysing the linear approximation of (18) around each stationary point. The Jacobian matrix for all points $(\phi, v)$ is

$$
J[F, G]_{(\phi, v)}=\left[\begin{array}{cc}
D^{\prime}(0) v & D(\phi) \\
-D^{\prime \prime}(0) v^{2}-g^{\prime}(\phi) & -c-2 D^{\prime}(\phi) v
\end{array}\right] .
$$

Evaluating (19) at $P_{0}$ we have

$$
J[F, G]_{(0,0)}=\left[\begin{array}{cc}
0 & 0 \\
-g^{\prime}(0) & -c
\end{array}\right],
$$

from which $\operatorname{tr} J[F, G]_{(0,0)}=-c<0$ and $\operatorname{det} J[F, G]_{(0,0)}=0$. Hence the linear system is inadequate to give us the local behaviour around $P_{0}$. Since the eigenvalues of (20) are $\lambda_{1}=0$ and $\lambda_{2}=-c, P_{0}$ is a non-hyperbolic point of codimension one (Arrowsmith and Place [5]). The corresponding eigenvectors are $v_{1}=\left(c,-g^{\prime}(0)\right)^{T}$ and $\boldsymbol{v}_{2}=(0,1)^{T}$, respectively.

Our non-linear local analysis uses the method of Andronov et al. [1] and the Centre Manifold Theorem. According with [1], in order to determine the phase portrait (18) around $P_{0}$ we are required to use higher order terms (h.o.t.) in the Taylor series. For (18) the second order terms are sufficient. The quadratic approximation to (18) around $P_{0}$ is

$$
\begin{aligned}
& \dot{\phi}=D^{\prime}(0) \phi v \equiv F_{2}(\phi, v) \\
& \dot{v}=-g^{\prime}(0) \phi-c v+G_{1}(\phi, v) \equiv G_{2}(\phi, v),
\end{aligned}
$$

where $G_{1}(\phi, v)=-\frac{1}{2} g^{\prime \prime}(0) \phi^{2}-D^{\prime}(0) v^{2}$. Therefore by the result in [1], the point $P_{0}$ is a saddle-node for the system (21) and hence for (18).

To complete the analysis around $P_{0}$ we have, by an application of the Centre Manifold Theorem (Carr [8]; Arrowsmith and Place [5]), that (21) has a unique one-dimensional invariant stable manifold locally tangent to the eigenvector $\boldsymbol{v}_{2}=(0,1)^{T}$ and a one-dimensional invariant centre manifold locally tangent to the eigenvector $v_{1}=\left(1,-g^{\prime}(0) / c\right)^{T}$. Both of these manifolds contain $P_{0}$. Moreover, Carr's theorems also guarantee that any trajectory of (21) in the vicinity of $P_{0}$, except those on the stable manifold, tend rapidly to the centre manifold. In other words, the dynamics around $P_{0}$ is given by the dynamics on the centre manifold.

For the application of the Centre Manifold Theorem we re-write the system (21) in its normal form, i.e., in the system of coordinates $\tilde{\phi}-\tilde{v}$ generated by the eigenvector basis $\left\{v_{1}, v_{2}\right\}$. To do this let us introduce the following notation

$$
\begin{gathered}
\boldsymbol{x}=\left[\begin{array}{l}
\phi \\
v
\end{array}\right], \quad \boldsymbol{y}=\left[\begin{array}{c}
\tilde{\phi} \\
\tilde{v}
\end{array}\right], \quad Q=\left[\begin{array}{cc}
1 & 0 \\
\frac{-g^{\prime}(0)}{c} & 1
\end{array}\right] \text { and } \\
\boldsymbol{f}(\boldsymbol{x})=\left[\begin{array}{c}
D^{\prime}(0) \phi v \\
\frac{-\boldsymbol{g}^{\prime}(0)}{2} \phi^{2}-D^{\prime}(0) v^{2}
\end{array}\right] .
\end{gathered}
$$


In the above terms the system (21) can be written as

$$
\dot{\boldsymbol{x}}=J[F, G]_{(0,0)} \boldsymbol{x}+\boldsymbol{f}(\boldsymbol{x}) .
$$

We define $\boldsymbol{y}$ such that $\boldsymbol{x}=Q \boldsymbol{y}$. Substituting into (22) we have

By noting that

$$
\dot{\boldsymbol{y}}=Q^{-1} J[F, G]_{(0,0)} Q y+\boldsymbol{f}(Q \boldsymbol{y}) .
$$

$$
Q^{-1} J[F, G]_{(0,0)} Q=\left[\begin{array}{cc}
0 & 0 \\
0 & -c
\end{array}\right]
$$

we write the normal form of (21) as follows

$$
\begin{aligned}
& \tilde{\phi}=-\frac{1}{c}\left[D^{\prime}(0) g^{\prime}(0)\right] \tilde{\phi}^{2}+D^{\prime}(0) \tilde{\phi}^{2} \tilde{v} \\
& \dot{v}=-c \tilde{v}-\frac{1}{2 c^{2}}\left[g^{\prime \prime}(0) c^{2}+4 D^{\prime}(0) g^{\prime 2}(0)\right] \tilde{\phi}^{2}+G_{3}(\tilde{\phi}, \tilde{v})
\end{aligned}
$$

where

$$
G_{3}(\tilde{\phi}, \tilde{v})=\frac{3 D^{\prime}(0) g^{\prime}(0)}{c} \tilde{\phi} \tilde{v}-D^{\prime}(0) \tilde{v}^{2}
$$

This is the standard form on which the centre manifold theory can be applied in a straightforward way. We denote by $\tilde{v}=h(\tilde{\phi})$ the unparametrized version of the centre manifold of (23).

Following Carr's theorems we have that the centre manifold for (23) can be approximated (up to second order) by (full details can be seen in SánchezGarduño [26] and Sánchez-Garduño and Maini [28]):

$$
h(\tilde{\phi})=-\left[\frac{g^{\prime \prime}(0) c^{2}+4 D^{\prime}(0) g^{\prime 2}(0)}{2 c^{3}}\right] \tilde{\phi}^{2}+O\left(\tilde{\phi}^{3}\right) .
$$

Therefore, in the original coordinates, denoting by $h$ (to avoid additional notation) the centre manifold for (21) (hence for (18)) we have

$$
h(\phi)=-\frac{g^{\prime}(0)}{c} \phi-\left[\frac{g^{\prime \prime}(0) c^{2}+4 D^{\prime}(0) g^{\prime 2}(0)}{2 c^{3}}\right] \phi^{2}+O\left(\phi^{3}\right) .
$$

The flow on the centre manifold of (21) is given by the equation

$\dot{w}=F_{2}(w, h(w))=-\frac{D^{\prime}(0)}{c}\left\{g^{\prime}(0) w^{2}+\left[\frac{g^{\prime \prime}(0) c^{2}+4 D^{\prime}(0) g^{\prime 2}(0)}{2 c^{2}}\right] w^{3}\right\}+O\left(\phi^{4}\right)$.

With functions $D$ and $g$ as in Sect. 3, Fig. 2 shows the dynamics given for the above equation for different values of $c$. There we have denoted by $s$ the ratio $\left[-4 D^{\prime}(0) g^{\prime 2}(0)\right] g^{\prime \prime}(0)$.

The above analysis shows that all trajectories of (21) with initial conditions $\left(\phi_{0}, v_{0}\right)$ such that $\sqrt{\phi_{0}^{2}+v_{0}^{2}}$ is very small:

1. tend to $P_{0}$ along the centre manifold for $\phi_{0}>0$,

2. move away from $P_{0}$ tending to the centre manifold for $\phi_{0}<0$. 


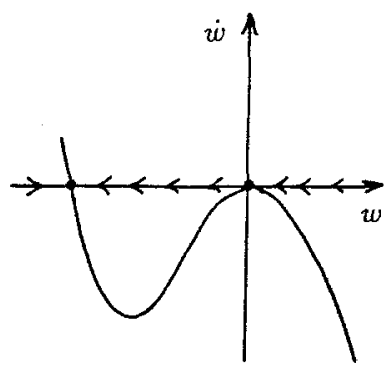

a.

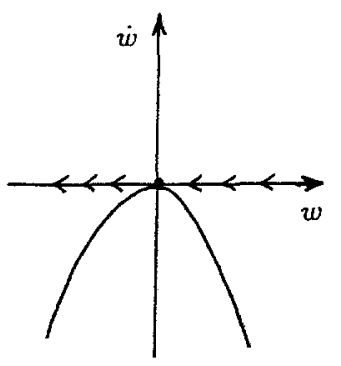

$b$

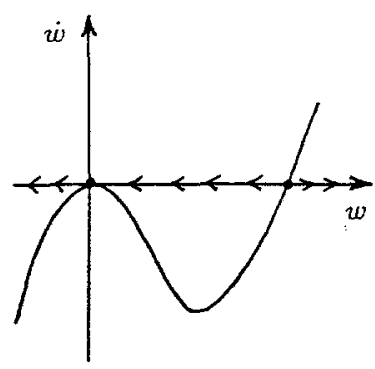

$c$

Fig. 2a-c. Dynamics on the centre manifold for the system (21) as the speed $c$ changes: a $c^{2}<s, \mathbf{b} c^{2}=s$ and $\mathbf{c} c^{2}>s$. Given that the equilibrium $w \equiv 0$ is unstable, the point $P_{0}$ is unstable too (see text for details)

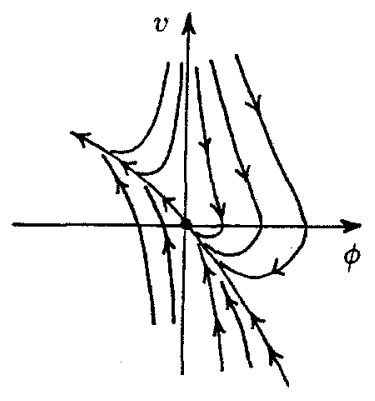

$a$

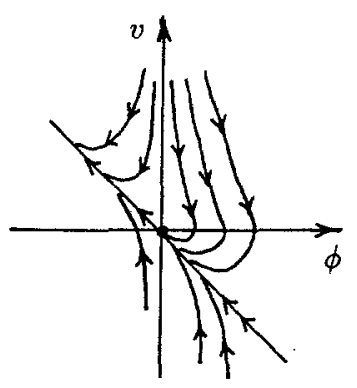

$b$

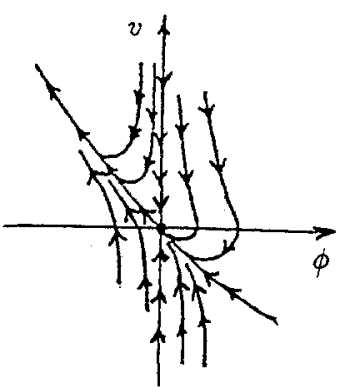

$c$

Fig. 3a-c. Local behaviour of the trajectories of (18) around the equilibrium $P_{0}$ for those values of $c$ as in Figs. 2a-c, respectively. This figure shows the centre manifold of the system (21), the nodal and saddle regions of $P_{0}$

Figure 3 shows the local behaviour of the trajectories of (21) (hence those of (18)) around the equilibrium $P_{0}$ for different values of $c$.

Now we complete the local analysis of the trajectories of (18). Evaluating (19) at $P_{1}$ we obtain

$$
J[F, G]_{(1,0)}=\left[\begin{array}{cc}
0 & D(1) \\
-g^{\prime}(1) & -c
\end{array}\right],
$$

from which it follows that $\operatorname{tr} J[F, G]_{(1,0)}=-c<0$ and $\operatorname{det} J[F, G]_{(1,0)}=$ $g^{\prime}(1) D(1)<0$. Thus $P_{1}$ is a saddle point. The roots of the characteristic polynomial of (24) are $\lambda_{1,2}=\left[-c \pm \sqrt{c^{2}-4 g^{\prime}(1) D(1)}\right] / 2$, with $\lambda_{1}>0$ and $\lambda_{2}<0$. The corresponding eigenvectors are $v_{1}=\left(1, \lambda_{1}\right)^{T}$ and $v_{2}=\left(1, \lambda_{2}\right)^{T}$, respectively.

The Jacobian matrix at $P_{c}$ is

$$
J[F, G]_{\left(0,-c / D^{\prime}(0)\right)}=\left[\begin{array}{cc}
-c & 0 \\
-\frac{D^{\prime \prime}(0) c^{2}}{\left(D^{\prime}(0)\right)^{2}}-g^{\prime}(0) & c
\end{array}\right] .
$$




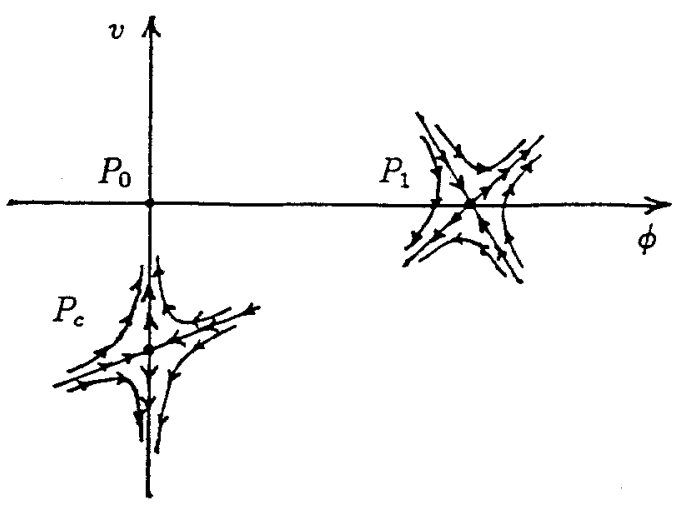

Fig. 4. Local phase portrait of (18) around the equilibria $P_{1}$ and $P_{c}$

Setting $v_{c}=-c / D^{\prime}(0)$, we have $\operatorname{tr} J[F, G]_{\left(0, v_{c}\right)}=0$ and $\operatorname{det} J[F, G]_{\left(0, v_{c}\right)}=$ $-c^{2}<0$. Hence $P_{c}$ is also a saddle point. The eigenvalues of (25) are $\lambda_{1}=-c$ and $\lambda_{2}=c$. The associated eigenvectors are $v_{1}=\left(1,-\frac{r}{2 c}\right)^{T}$ and $v_{2}=(0,1)^{T}$, where $r=-\frac{D^{\prime \prime}(0) c^{2}}{\left(D^{\prime}(0)\right)^{2}}-g^{\prime}(0)$.

The phase portrait of (18) around $P_{1}$ and $P_{c}$ is as shown in Fig. 4.

In the following sections we extend this local analysis to the whole phase portrait of (18) to obtain the t.w.s. dynamics of (10).

\section{Global analysis I: the existence of a continuum of front type solutions}

Given the equivalence of searching for t.w.s. for (10) and looking for heteroclinic trajectories of the corresponding ODE system in travelling wave coordinates, we focus our attention on finding values of the speed $c$ for which there exist trajectories of (18) connecting pairs of equilibrium points.

First, we introduce the following notation: Let $W_{c}^{u}\left(P_{1}\right)$ and $W_{c}^{s}\left(P_{c}\right)$ be the left-unstable manifold of $P_{1}$ and the right-stable manifold of $P_{c}$, respectively. The following proposition holds:

Proposition 2. For $c=0$ the system (18) is hamiltonian-like. Moreover $W_{0}^{u}\left(P_{1}\right)$ tends to $(0,-\infty)$ as $\tau \rightarrow+\infty$. The phase portrait is as illustrated in Fig. $5 a$.

Proof. For $c=0$ the system (18) becomes

$$
\begin{aligned}
\dot{\phi} & =D(\phi) v \\
\dot{v} & =-D^{\prime}(\phi) v^{2}-g(\phi) .
\end{aligned}
$$

Now we construct the system

$$
\begin{aligned}
\dot{\phi} & =[D(\phi)]^{2} v \\
\dot{v} & =-D(\phi) D^{\prime}(\phi) v^{2}-D(\phi) g(\phi) .
\end{aligned}
$$



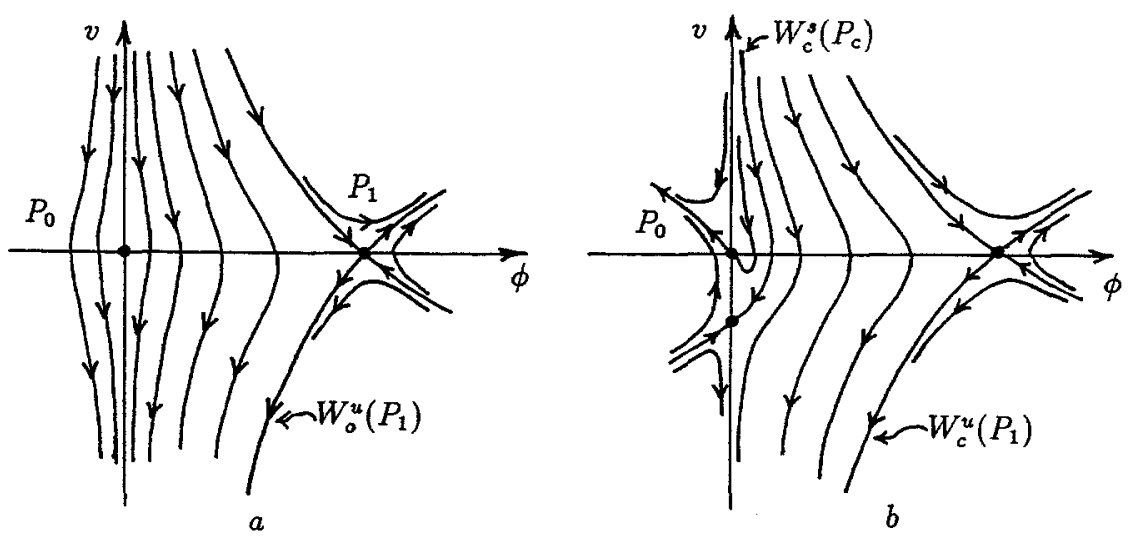

Fig. 5.a, b. Phase portrait of the system (18) for small values of $c$ : $\mathbf{a} c=0$ and $\mathbf{b}$ sufficiently small positive values of $c$

Since $D(\phi)>0 \forall \phi \in(0,1]$ and is a well behaved function there, the paths of the trajectories of (26) and (27) coincide on the region $\mathscr{R}$ defined as follows

$$
\mathscr{R}=\{(\phi, v) \mid 0<\phi \leqq 1,-\infty<v<+\infty\} .
$$

Note that (27) can be written in the hamiltonian form

$$
\begin{aligned}
& \dot{\phi}=\frac{\partial H}{\partial v} \\
& \dot{v}=-\frac{\partial H}{\partial \phi}
\end{aligned}
$$

where

$$
H(\phi, v)=\frac{1}{2}[D(\phi) v]^{2}+\int_{\phi_{0}}^{\phi} D(s) g(s) d s,
$$

with $\phi_{0} \in(0,1)$. The trajectories of (27) (and thus those of (26)) coincide with the level curves of $H(\phi, v) \equiv C_{1} \in \boldsymbol{R}$. In particular, the trace (path) of the trajectory of (26) passing through $(1,0)$ is given by the level curve

$$
\frac{1}{2}[D(\phi) v]^{2}+\int_{\phi_{0}}^{\phi} D(s) g(s) d s=H(1,0),
$$

where

$$
H(1,0)=\int_{\phi_{0}}^{1} D(s) g(s) d s .
$$

Hence the negative branch is given by

$$
v(\phi)=\frac{-1}{D(\phi)} \sqrt{2\left[H(1,0)-\int_{\phi_{0}}^{\phi} D(s) g(s) d s\right]} .
$$


That the function $v$ is well defined on $(0,1]$ can be seen by setting $\mathscr{D}(\phi)=\int_{\phi_{0}}^{\phi} D(s) g(s) d s$ and verifying that: $\left.\left.i\right) \mathscr{D}(\phi)>0 \forall \phi \in(0,1], i i\right) \mathscr{D}$ is strictly increasing on $\left(\phi_{0}, 1\right)$ iii) $\mathscr{D}$ attains its maximum at $\phi=1$, which is $\mathscr{D}(1)=H(1,0)$.

From the explicit form of the path of $W_{o}^{u}\left(P_{1}\right)$ given in the above formula it can be seen that $\lim _{\phi \rightarrow 0^{+}} v(\phi)=-\infty$. This completes the proof.

To continue our analysis we must use the continuity property of the solutions of (18) with respect to the parameter $c$. Therefore we state the following result:

Proposition 3. The solution $(\phi(\tau), v(\tau))$ of the initial value problem

$$
\begin{aligned}
& \dot{\phi}=D(\phi) v \\
& \dot{v}=-c v-D^{\prime}(\phi) v^{2}-g(\phi)
\end{aligned}
$$

with $\phi\left(\tau_{0}\right)=\phi_{0}, v\left(\tau_{0}\right)=v_{0}$ depends continuously on $\tau_{0}, \phi_{0}, v_{0}$ and $c$.

Proof. Given that the vector field defined by (18) depends differentiably on $\phi$, $v$ and $c$, the proof follows by applying the corresponding general theorem on continuity of solutions with respect to the parameters and initial conditions. Full details can be found in Arnold [2].

The following proposition can be proved:

Proposition 4. For sufficiently small positive values of the speed $(c \approx 0)$, the path $v(\phi)$ of $W_{c}^{u}\left(P_{1}\right)$ tends to $-\infty$ as $\phi \rightarrow 0^{+}$, while $W_{c}^{s}\left(P_{c}\right)$ leaves (in inverse time) the region

$$
\mathscr{R}=\{(\phi, v) \mid 0<\phi \leqq 1,-\infty<v<0\}
$$

at some point $\phi_{0}$ situated to the right of $P_{0}$ but sufficiently close to $P_{0}$. The phase portrait is illustrated in Fig $5 b$.

Proof. The first part follows by using Propositions 2 and 3. For the second part we have that for a given $c$, the uniqueness property implies that the manifolds $W_{c}^{u}\left(P_{1}\right)$ and $W_{c}^{s}\left(P_{c}\right)$ cannot cross each other. Now $W_{c}^{u}\left(P_{1}\right) \rightarrow(0,-\infty)$ as $\tau \rightarrow \infty$ and for small values of $c$ the vector field (18) behaves as in Fig. 5, hence $W_{c}^{s}\left(P_{c}\right)$ must leave (in inverse time) the region $\mathscr{R}$ on the interval $(0,1)$. Because of the position of $P_{c}$ for small $c$ and the vertical null-clines of (18) (see Fig. 9), the exit point must be close to $P_{0}$.

Remark 1. Note that for $c \neq 0$ the equilibria $P_{c}$ and $P_{0}$ are always connected by a trajectory (a trivial heteroclinic trajectory), but associated with this trajectory we have $\phi \equiv 0$ which, from the physical interpretation of $\phi$, is not important. However, the non-existence of any heteroclinic trajectory of the system (18) for sufficiently small values of $c$ implies the non-existence of t.w.s. for equation (10).

In the next part of our analysis, the role of the vertical null-clines (18) will be important. From (18), $\dot{v}=0$ only on the graph of the following 
two functions:

$$
V_{1}(\phi)=\frac{-c+\sqrt{c^{2}-4 D^{\prime}(\phi) g(\phi)}}{2 D^{\prime}(\phi)}
$$

and

$$
V_{2}(\phi)=\frac{-c-\sqrt{c^{2}-4 D^{\prime}(\phi) g(\phi)}}{2 D^{\prime}(\phi)}
$$

Let us list some properties of $V_{1}$ and $V_{2}$ valid for all positive values of $c$ :

1. $V_{1}(0)=V_{1}(1)=0, V_{2}(0)=-\frac{c}{D^{\prime}(0)}$ and $V_{2}(1)=-\frac{c}{D^{\prime}(1)}$,

2. As $V_{1}^{\prime}(0)=-\frac{g^{\prime}(0)}{c}<0$ and $V_{1}^{\prime}(1)=-\frac{g^{\prime}(1)}{c}>0$, by the Mean Value The3. orem, there exists a point $\tilde{\phi} \in(0,1)$ such that $V_{1}^{\prime}(\widetilde{\phi})=0$,

$$
V_{2}^{\prime}(0)=\frac{D^{\prime 2}(0) g^{\prime}(0)+c^{2} D^{\prime \prime}(0)}{D^{\prime 2}(0) c}
$$

and

$$
V_{2}^{\prime}(1)=\frac{D^{\prime 2}(1) g^{\prime}(1)+c^{2} D^{\prime \prime}(1)}{D^{\prime 2}(1) c}
$$

Note that the signs of both $V_{2}^{\prime}(0)$ and $V_{2}^{\prime}(1)$ depend on the sign of the numerators in (33) and (34), respectively, which, in turn, depends on the local profile (at $\phi=0$ and $\phi=1$ ) of the diffusion coefficient $D$. Here we will distinguish the following cases:

1. $D^{\prime \prime}(\phi)>0 \forall \phi \in[0,1]$,

2. $D^{\prime \prime}(\phi)<0 \forall \phi \in[0,1]$.

Observe that the condition on $D$ to be a strictly increasing function on $[0,1]$, together with the above condition 1 . implies $D^{\prime}(0)<D^{\prime}(\phi)$ on $(0,1]$; while from condition 2. given above, together with assumptions 3 and 4 in Sect. 3 , we have $D^{\prime}(0)>D^{\prime}(\phi)$ on the same interval.

Firstly let us determine the sign of $V_{2}^{\prime}(0)$ for each of the above two cases: if $D^{\prime \prime}(0)>0$, then $V_{2}^{\prime}(0)>0$, while for $D^{\prime \prime}(0)<0$ we have the following possibilities:

1. $c^{2}<-\frac{g^{\prime}(0) D^{\prime 2}(0)}{D^{\prime \prime}(0)} \Rightarrow V_{2}^{\prime}(0)>0$,

2. $c^{2}=-\frac{g^{\prime}(0) D^{\prime 2}(0)}{D^{\prime \prime}(0)} \Rightarrow V_{2}^{\prime}(0)=0$,

3. $c^{2}>-\frac{g^{\prime}(0) D^{\prime 2}(0)}{D^{\prime \prime}(0)} \Rightarrow V_{2}^{\prime}(0)<0$. 
We have analogous properties for $V_{2}^{\prime}(1)$. If $D^{\prime \prime}(0)<0$ then $V_{2}^{\prime}(1)<0$, while for $D^{\prime \prime}(0)>0$ we have the following possibilities:

1. $c^{2}<-\frac{g^{\prime}(1) D^{\prime 2}(1)}{D^{\prime \prime}(1)} \Rightarrow V_{2}^{\prime}(1)>0$,

2. $c^{2}=-\frac{g^{\prime}(1) D^{\prime 2}(1)}{D^{\prime \prime}(1)} \Rightarrow V_{2}^{\prime}(1)=0$,

3. $c^{2}>-\frac{g^{\prime}(1) D^{\prime 2}(1)}{D^{\prime \prime}(1)} \Rightarrow V_{2}^{\prime}(1)<0$.

In order to consider precisely the behaviour of $V_{1}$ and $V_{2}$ on $[0,1]$ let us define the function $\mathscr{F}$ by $\mathscr{F}(\phi)=4 D^{\prime}(\phi) g(\phi)$. Since $D$ and $g$ are well behaved functions on $[0,1], \mathscr{F}$ attains its maximum there. Let us denote it by $M$. Figure 6 illustrates typical shapes of $\mathscr{F}$.

The shapes of $V_{1}$ and $V_{2}$ also depend on the values of $c$ compared with $M$. We consider the following two cases:

Case 1. $c^{2} \geqq M$. Here we have:

1. The domain of $V_{1}$ and $V_{2}$ is the interval $[0,1]$,

2. $V_{1}(\phi) \leqq 0$ and $V_{2}(\phi)<0 \forall \phi \in[0,1]$, with $V_{1}(\phi) \geqq V_{2}(\phi)$; the equality occurs at $\phi^{*} \in(0,1)$ such that $c^{2}=M=\mathscr{F}\left(\phi^{*}\right)$, and the common value then is $-\sqrt{\frac{g\left(\phi^{*}\right)}{D^{\prime}\left(\phi^{*}\right)}}$, where $\phi^{*}$ is the point at which the function $\mathscr{F}$ attains its maximum,

3. Given that $\mathscr{F}(\phi)$ is a well behaved (bounded) function on $[0,1]$, it follows that for sufficiently large $c, V_{1}(\phi)$ tends to the horizontal axis and $V_{2}(\phi)$ becomes more negative on $(0,1)$.
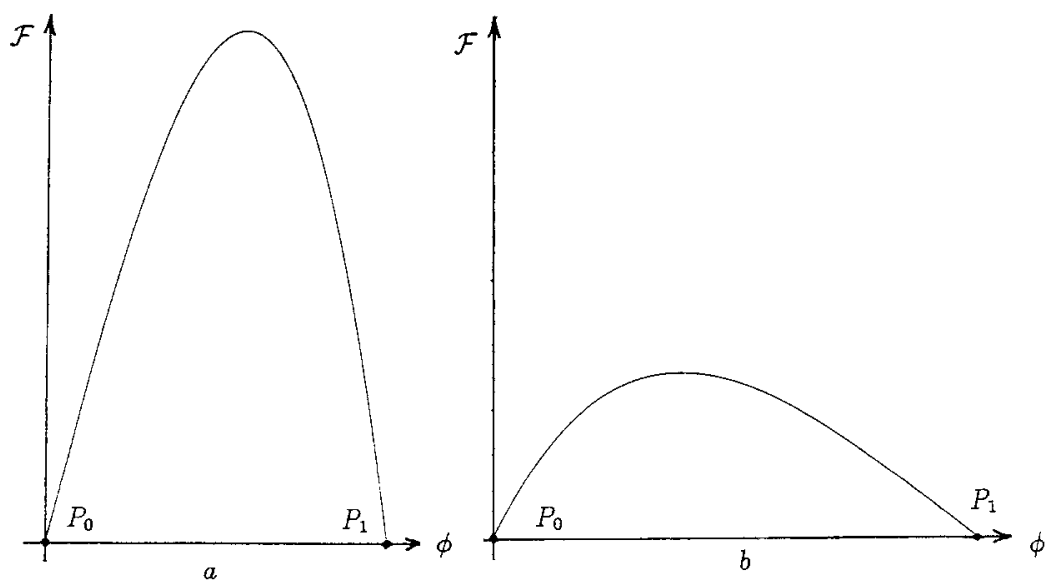

Fig. 6a, b. For functions $D$ and $g$ as in Sect. 3 these are typical behaviours of $\mathscr{F}(\phi)=4 D^{\prime}(\phi) g(\phi)$ on $[0,1]$. a For $D(\phi)=\left(2 \phi+\phi^{2}\right)$ and $g(\phi)=\phi(1-\phi)$; b For $D(\phi)=(1-\exp (-\phi))$ and $g(\phi)=\phi(1-\phi)$ 
Figures 7 and 8 show the typical behaviour of the vertical null-clines defined by (18) corresponding to the geometrical features of $D$ and $g$ mentioned in Sect. 3, together with the vector field defined by (18).

Case 2. $c^{2}<M$. Here the domain of $V_{1}$ and $V_{2}$ is the union of at least two disjoint sub-intervals contained in $[0,1]$. The qualitative behaviour of $V_{1}$ and $V_{2}$ where they are defined is illustrated in Fig. 9.

Now we determine the way in which $W_{c}^{u}\left(P_{1}\right)$ leaves $P_{1}$ and $W_{c}^{s}\left(P_{\mathrm{c}}\right)$ attains the point $P_{c}$. The local analysis around $P_{1}$ and $P_{c}$ gives us the following information: The slope, $M_{c}^{u}\left(P_{1}\right)$, of $W_{c}^{u}\left(P_{1}\right)$ at $P_{1}$ is given by

$$
M_{c}^{u}\left(P_{1}\right)=\frac{-c+\sqrt{c^{2}-4 D(1) g^{\prime}(1)}}{2 D(1)}
$$
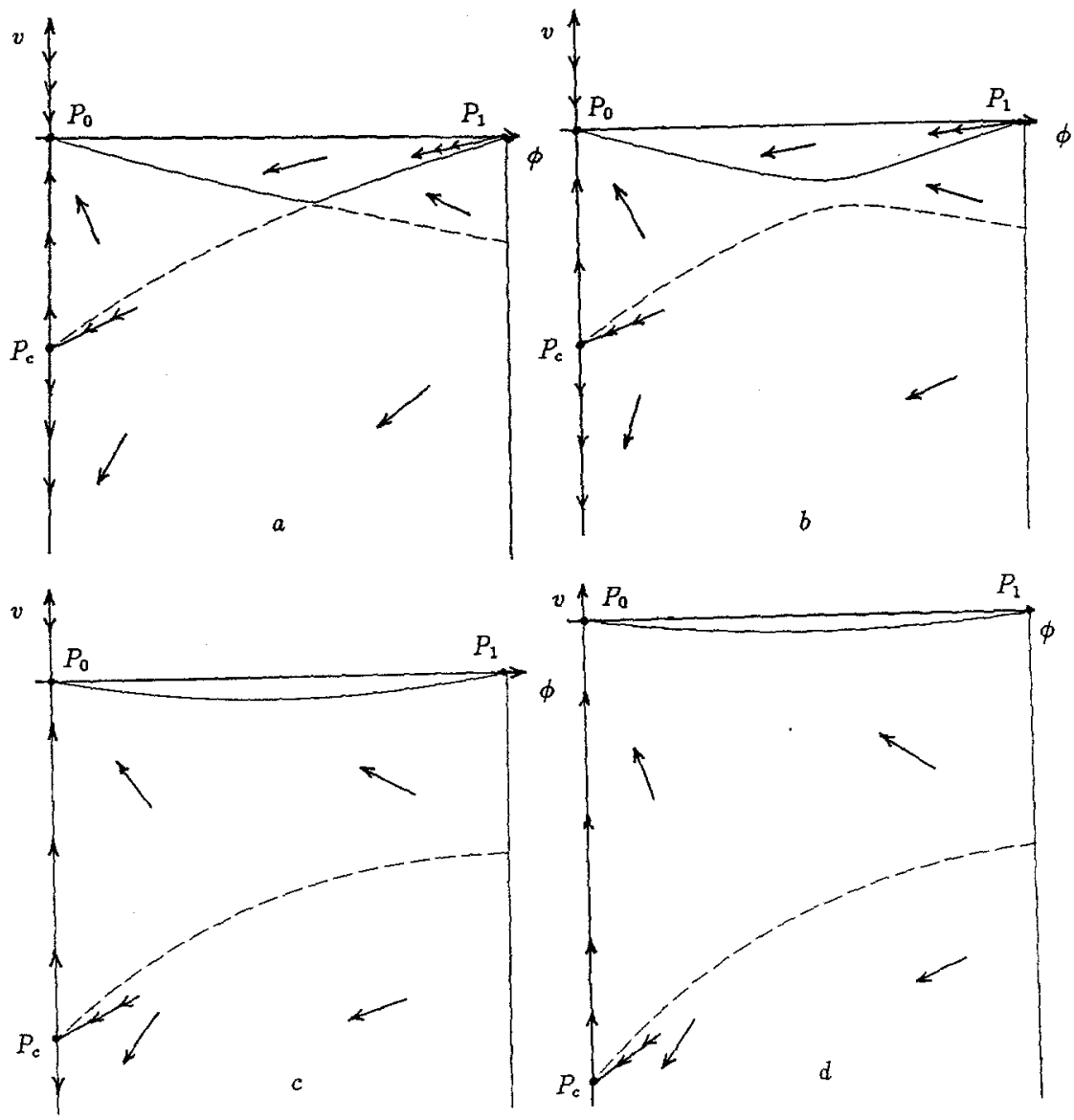

Fig. 7a-d. Vertical null-clines of (18) for $c^{2} \geqq M$ with $D(\phi)=2 \phi+\phi$ and $g(\phi)=\phi(1-\phi)$. As $D^{\prime \prime}(0)>0$, it follows that $V_{2}^{\prime}(0)$ and $V_{2}^{\prime}(1)$ changes sign: see text for details. a $c^{2}=M$. For $c^{2}>M$ : b $V_{2}^{\prime}(1)<0$, $\mathbf{c} V_{2}^{\prime}(1)=0$ and $\mathbf{d} V_{2}^{\prime}(1)>0$. The continuous line represents $V_{1}$ and the broken line $V_{2}$ 

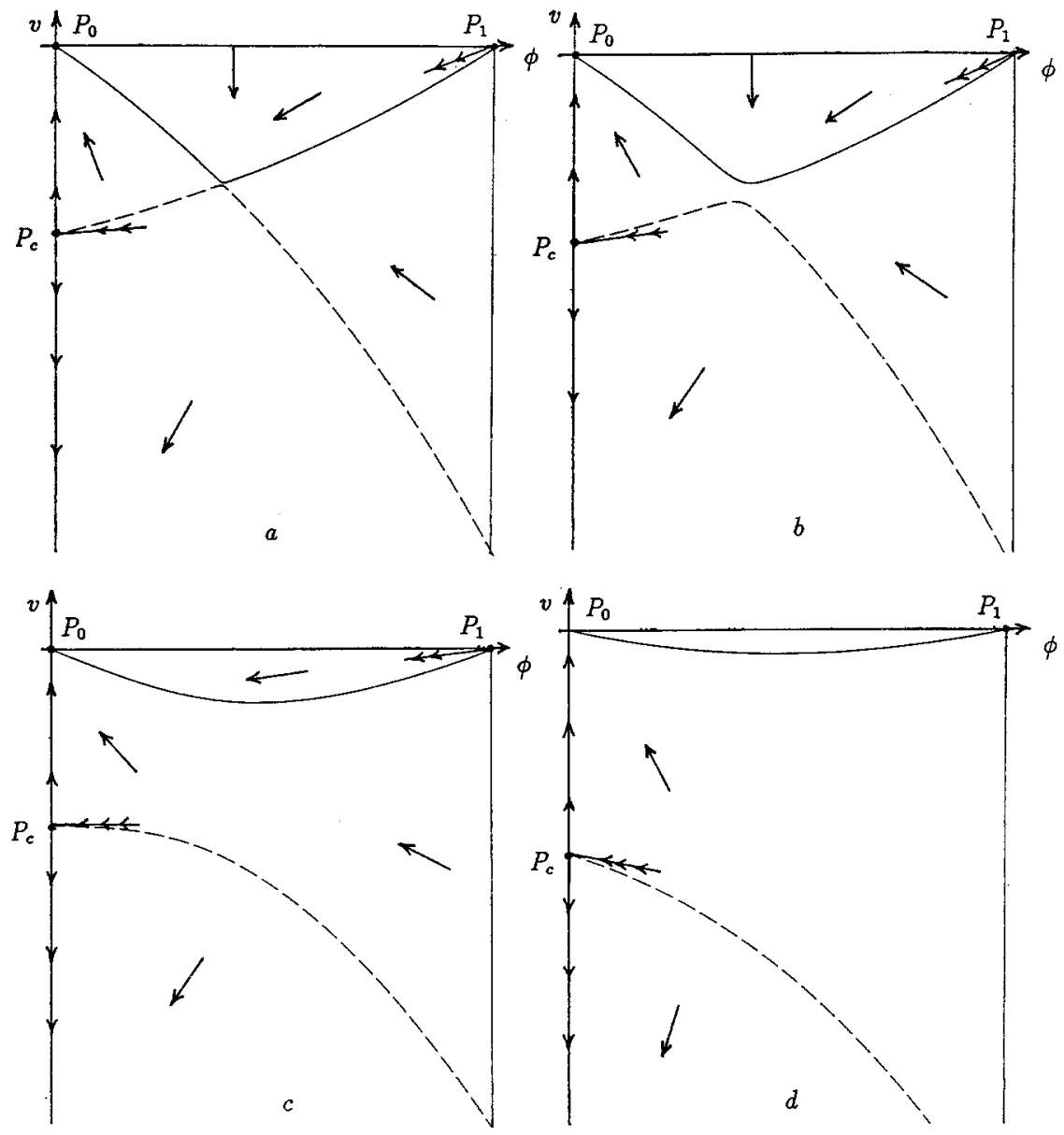

Fig. 8a-d. Vertical null-clines of (18) for $c^{2} \geqq M$ with $D(\phi)=1-\exp (-\phi)$ and $g(\phi)=\phi(1-\phi)$. As $D^{\prime \prime}(0)<0$, it follows that $V_{2}^{\prime}(1)<0$ and $V_{2}^{\prime}(0)$ changes its sign depending on $c$ : see text for details. a $c^{2}=M$. For $c^{2}>M$ : b $V_{2}^{\prime}(0)<0$, c $V_{2}^{\prime}(0)=0$ and d $V_{2}^{\prime}(0)>0$. The continuous line represents $V_{1}$ and the broken line $V_{2}$

and the slope, $M_{c}^{s}\left(P_{c}\right)$, of $W_{c}^{s}\left(P_{c}\right)$ at $P_{c}$ is given by

$$
M_{c}^{s}\left(P_{c}\right)=\frac{D^{\prime 2}(0) g^{\prime}(0)+c^{2} D^{\prime \prime}(0)}{2 D^{\prime 2}(0) c} .
$$

We now compare $V_{1}^{\prime}(1)$ with $M_{c}^{u}\left(P_{1}\right)$ and $V_{2}^{\prime}(0)$ with $M_{c}^{s}\left(P_{c}\right)$, respectively. For given $D$ and $g$, Fig. 10 shows the behaviour of $V_{1}^{\prime}(1)$ and of $M_{c}^{u}\left(P_{1}\right)$, both as functions of $c$.

Comparing directly (33) with (36) we conclude that $M_{c}^{s}\left(P_{c}\right)<V_{2}^{\prime}(0)$ for all $c$ provided both are positive. The inequality is reversed when both are negative. Figure 11 shows how $W_{c}^{u}\left(P_{1}\right)$ leaves $P_{1}$ and how $W_{c}^{s}\left(P_{c}\right)$ approaches $P_{c}$. It illustrates the local behaviour of the vertical null-clines of (18) at $P_{1}$ and at $P_{c}$. 


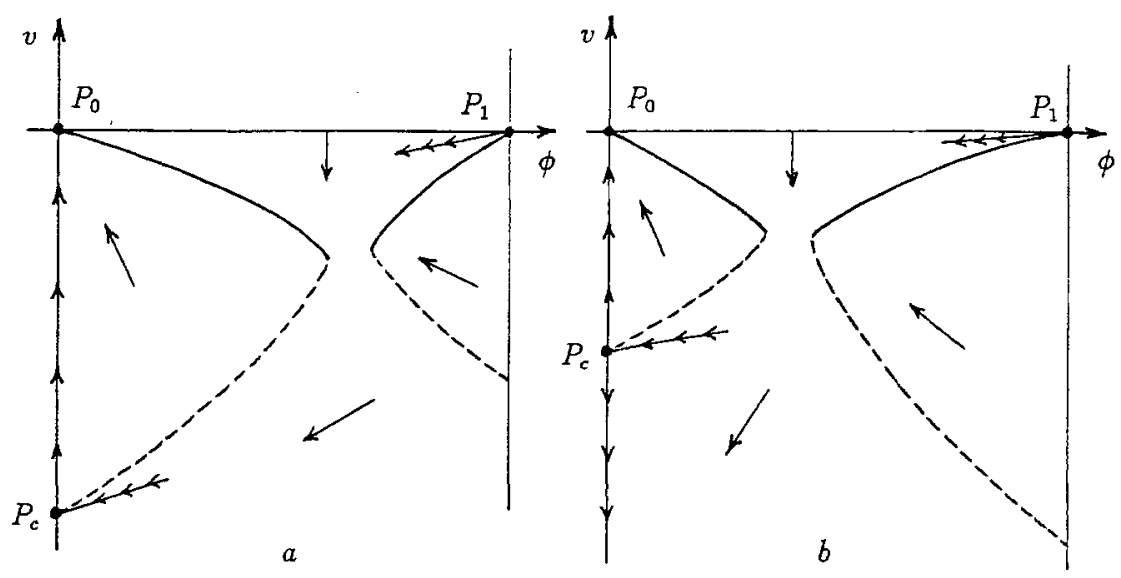

Fig. 9a, b. Qualitative behaviour of $V_{1}$ and $V_{2}$ for $c^{2}<M$ with $D$ and $g$ as in: a Fig. 7 and b Fig. 8

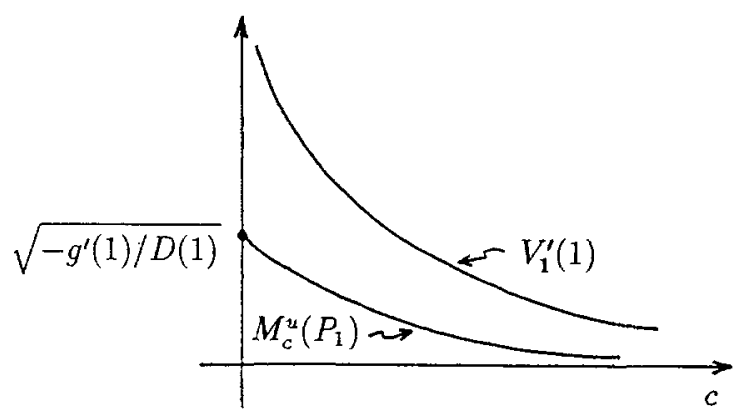

Fig. 10. Slopes of the vertical null-cline of (18) and of $W_{c}^{u}\left(P_{1}\right)$ at $\phi=1$, as functions of $c$

With all this information, we are able to prove the following lemma:

Lemma 1. For each $c$ such that $\left.c^{2} \geqq M=\max 4 D^{\prime}(\phi) g(\phi)\right]$ where the maximum is taken on $[0,1]$, there exists a heteroclinic trajectory of $(18)$ connecting $P_{1}$ with $P_{0}$. Moreover, the trajectory $W_{c}^{s}\left(P_{c}\right)$ leaves (in reverse time) the region

$$
\mathscr{R}=\{(\phi, v) \mid 0<\phi \leqq 1,-\infty<v<0\}
$$

somewhere on $\{(\phi, v) \mid \phi=1, v<0\}$.

Proof. This follows from using the vector field defined by (18), the vertical nullclines of (18) and the local analysis of (25) at $P_{1}$ and $P_{c}$.

In both cases $D^{\prime \prime}(\phi)>0$ and $D^{\prime \prime}(\phi)<0 \forall \phi \in[0,1]$ with $c^{2} \geqq M$, we have two types of vertical null-clines: those with a common point, or those without any intersection at all, as can be seen from Figs. 7 and 8 . Let us start with the situation as in Fig. 12a i.e., with the vertical null-clines as in Fig. 8a. The unstable manifold $W_{c}^{u}\left(P_{1}\right)$ leaves $P_{1}$ as shown in Fig. 12a and enters the region 

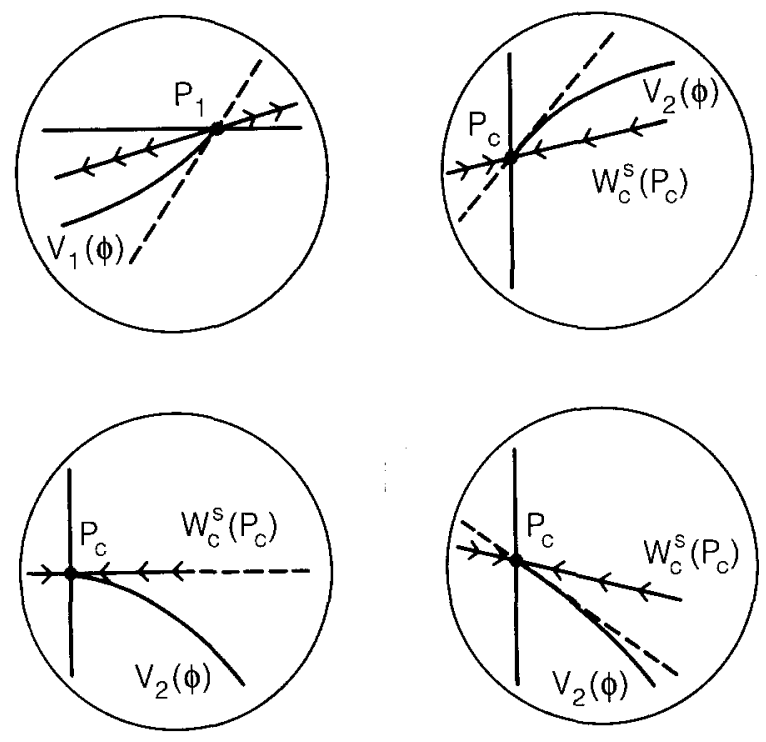

Fig. 11. Behaviour of $W_{c}^{u}\left(P_{1}\right)$ at $P_{1}$ and that of $W_{c}^{s}\left(P_{c}\right)$ at $P_{c}$, for different values of $c$ (see text for details). Compare their behaviour to that $V_{1}(\phi)$ and $V_{2}(\phi)$ at the same points
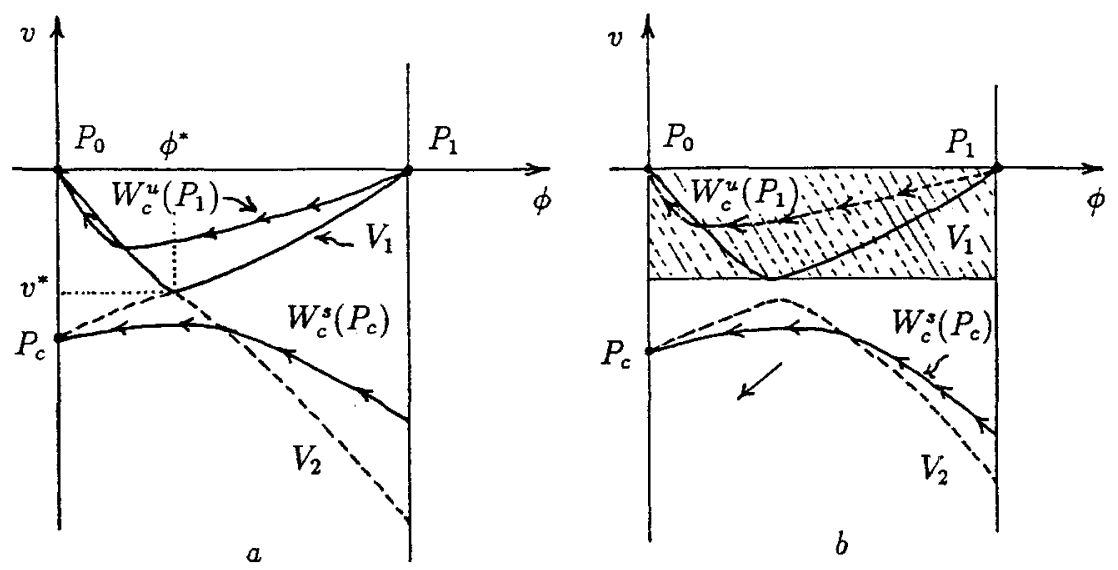

Fig. 12a, b. Proof of Lemma 1, for: a vertical null-clines as in Fig. 7a. b vertical null-clines as in Fig. 7b. See text for details

$\mathscr{R}_{2}=\left\{(\phi, v) \mid 0<\phi<1, V_{1}(\phi) \leqq v<0\right\}$, where the vector field pushes it towards the graph of $V_{1}$. Let $\left(\phi_{0}, V_{1}\left(\phi_{0}\right)\right)$ be the first intersection of $W_{c}^{u}\left(P_{1}\right)$ with the graph of $V_{1}$. If $\phi_{0}>\phi^{*}$, then $W_{c}^{u}\left(P_{1}\right)$ re-enters the region $\mathscr{R}_{2}$ and eventually re-attains $V_{1}$. If this occurs for $\phi<\phi^{*}$, then the vector field pushes $W_{c}^{u}\left(P_{1}\right)$ towards $P_{0}$, while if $\phi_{0} \leqq \phi^{*}$ then there are no more intersecting points and $W_{c}^{u}\left(P_{1}\right)$ reaches $P_{0}$ as time tends to $+\infty$. 
However, $W_{c}^{s}\left(P_{c}\right)$ reaches $P_{c}$ as can be seen in Fig. 12a. This occurs because with this behaviour of the vertical null-clines the unique possibility for $W_{c}^{s}\left(P_{c}\right)$ to intersect the graph of $V_{2}$ is at the point $\left(\phi_{1}, V_{2}\left(\phi_{1}\right)\right)$ with $\phi_{1}>\phi^{*}$. In this situation, for inverse time, $W_{c}^{s}\left(P_{c}\right)$ must leave the region $\mathscr{R}_{2}$ at the point $Q=(1, v)$ where $V_{2}(1)<v<0$.

For the case in Fig. $12 \mathrm{~b}$ (which corresponds to Fig. $8 \mathrm{~b}$ ) we conclude that $W_{c}^{u}\left(P_{1}\right)$ ends at $P_{0}$ by showing that the shaded region is a positive invariant set of (18) and by using the Poincaré-Bendixon Theorem. We can follow similar arguments as those given in the case of Fig. 12a to prove that $W_{c}^{s}\left(P_{c}\right)$ has the same qualitative behaviour. This completes the proof.

To summarise the analysis carried out thus far, we have proved the following theorem:

Theorem 1. If the functions $D$ and $g$ satisfy the conditions 1-4 in Sect. 1 and for $M$ as defined above, then (10):

1. Has no t.w.s. for sufficiently small $c$,

2. Possesses a monotone decreasing travelling wave solution of front type $u(x, t)=\phi(x-c t)$ satisfying the boundary conditions $\phi(-\infty)=1$ and $\phi(+\infty)=0$, for each $c$ such that $c^{2} \geqq M=\max \left[4 D^{\prime}(\phi) g(\phi)\right]$.

\section{Global analysis II: monotonicity properties and the existence of a sharp type solution}

In the previous section, we proved the non-existence of t.w.s. for (1) for sufficiently small $c$. We also stated there the existence of a front type solution for each $c$ such that $c^{2} \geqq M=\max \left[4 D^{\prime}(\phi) g(\phi)\right]$. In this section we investigate the phase portrait of (18) (thus the possibility of existence of t.w.s. for (10)) for $c \in I_{M} \equiv\left[c_{0}, \sqrt{M}\right]$, where $c_{0}$ is a positive and sufficiently small value of $c$.

Let $c_{1}$ and $c_{2}$ be two arbitrary values of the speed on $I_{M}$ with $c_{1}<c_{2}$. Define $v_{c_{1}}(\phi)$ and $v_{c_{2}}(\phi)$ as the trace (path) of $W_{c_{1}}^{s}\left(P_{c_{1}}\right)$ and $W_{c_{2}}^{s}\left(P_{c_{2}}\right)$, respectively, whose graphs belong to the region $\mathscr{R}=\{(\phi, v) \mid 0 \leqq \phi \leqq 1, v \leqq 0\}$. In a similar way define $v_{c_{0}}(\phi)$ and $v_{\sqrt{M}}(\phi)$. We can prove our first monotonicity result:

Proposition 5. For all $\phi \in[0,1]$ the following inequality holds

$$
v_{\sqrt{M}}(\phi)<v_{c_{2}}(\phi)<v_{c_{1}}(\phi)<v_{c_{0}}(\phi) \text {. }
$$

Proof. Note that the equilibrium $P_{c}=\left(0,-c / D^{\prime}(0)\right)$ moves monotonically away from $P_{0}$ on the negative vertical axis as $c$ increases. Since $\frac{\partial}{\partial c}(\dot{v})=-v>0$, we have that the vertical component of the vector field defined by (18) is an increasing function $c$ on the region $\mathscr{R}$ defined above, while the horizontal component does not change with $c$. 
Given that the argument is the same for any pair of functions $v$ in (37) we prove the inequality only for $v_{c_{0}}$ and $v_{c_{1}}$. Let us define the region $\mathscr{R}_{0}$ as (see Fig. 13):

$$
\mathscr{R}_{0}=\left\{(\phi, v) \mid 0 \leqq \phi \leqq \phi_{0}, v_{c_{0}}(\phi) \leqq v \leqq 0\right\}
$$

Let $\left(\bar{\phi}, v_{\mathcal{c}_{0}}(\bar{\phi})\right)$ be any point on that part of the boundary of $\mathscr{R}_{0}$ on the graph of $v_{c_{0}}(\phi)$. We now consider the vector field (18) for $c=c_{1}$. For the above property of $(\dot{\phi}, \dot{v})$ with respect to $c$, each trajectory through $\left(\bar{\phi}, v_{c_{0}}(\bar{\phi})\right)$ enters $\mathscr{R}_{0}$ and ends at $P_{0}$ when $\tau \rightarrow+\infty$. For $c=c_{1}, v_{c_{1}}(0)=-c_{1} / D^{\prime}(0)<-c_{0} / D^{\prime}(0)$, thus the point $\left(0,-c_{1} / D^{\prime}(0)\right)$ is outside of $\mathscr{R}_{0}$ and $W_{c_{1}}^{s}\left(P_{1}\right)$ cannot enter $\mathscr{R}_{0}$ in reverse time. Hence the result follows.

Remark 2. By Proposition 5 we have that if $W_{c_{1}}^{s}\left(P_{c_{1}}\right)$ and $W_{c_{2}}^{s}\left(P_{c_{2}}\right)$ leave $\mathscr{R}$ on $(0,1)$, then $W_{c_{2}}^{s}\left(P_{c_{2}}\right)$ does so to the right of $W_{c_{1}}^{s}\left(P_{c_{1}}\right)$, while if both leave $\mathscr{R}$ on $\{(\phi, v) \mid \phi=1, v<0\}$, then $W_{c_{2}}^{s}\left(P_{c_{2}}\right)$ does so below $W_{c_{1}}^{s}\left(P_{c_{1}}\right)$.

To state our second result on monotonicity we start by writing the first order ODE for the trajectories of (18):

$$
\frac{d v}{d \phi}=\frac{-c v-D^{\prime}(\phi) v^{2}-g(\phi)}{D(\phi) v}
$$

and note that $v=v(\phi)$ also satisfies the boundary condition $v(1)=0$.

Now we define the function $\mathscr{G}(\phi)$ as

$$
\mathscr{G}(\phi)=\left(v_{2}-v_{1}\right) \exp \left\{-\int_{\tilde{\phi}}^{\phi} \frac{\left(v_{1}^{2} v_{2}-v_{2}^{2} v_{1}\right) D^{\prime}(s)+g(s)\left(v_{2}-v_{1}\right)}{v_{1} v_{2}\left(v_{2}-v_{1}\right) D(s)} d s\right\}
$$

where $\tilde{\phi} \in(0,1)$ with $\tilde{\phi}<\phi$.

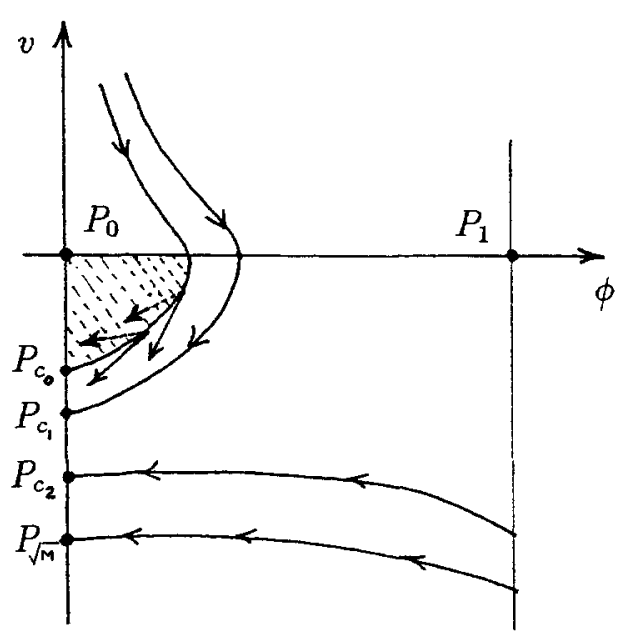

Fig. 13. Proof of Proposition 5. See text for details 
Given that the trajectories of (18) leaving $P_{1}$ enter the region $\mathscr{R}$, and in the extremum case (for large $c$ ) end at $P_{0}$, it makes sense to consider that both $v_{1}$ and $v_{2}$ are negative on $(0,1)$. The following proposition holds:

Proposition 6. Let $v_{1}$ and $v_{2}$ be two solutions of (38) corresponding to the speeds $c_{1}$ and $c_{2}$, respectively, satisfying: i) $v_{1}(\mathbf{1})=v_{2}(1)=0$ and ii) $v_{1}(\phi)<0$ and $v_{2}(\phi)<0 \forall \phi \in(0,1)$. Then $\mathscr{G}(\phi) \rightarrow 0$ when $\phi \rightarrow 1$.

Proof. This can be seen in $[27,28]$

The following proposition gives us a monotonicity property of the solutions of equation (38) with respect to the speed $c$.

Proposition 7. Let $v_{1}(\phi)$ and $v_{2}(\phi)$ be two solutions of (38) corresponding to $c_{1}$ and $c_{2}$, respectively. Suppose that $v_{1}$ and $v_{2}$ satisfy; a) $v_{1}(1)=v_{2}(1)=0$ and b) $\left[v_{1}(\phi) v_{2}(\phi)\right]>0 \forall \phi \in(0,1)$. Then for all $\phi$ in the interval $(0,1)$ :

1. $v_{1}(\phi)=v_{2}(\phi)$ if $c_{1}=c_{2}$,

2. $v_{1}(\phi)>v_{2}(\phi)$ if $c_{1}>c_{2}$

3. $v_{1}(\phi)<v_{2}(\phi)$ if $c_{1}<c_{2}$.

Proof. This follows by using Proposition 6. Full details can be seen in [27].

Remark 3. In geometrical terms, Proposition 7 gives us the relative position of the graphs of $v_{1}$ and $v_{2}$ for $\phi \in(0,1)$. For example, if $c_{1}>c_{2}$ (both positive) then the corresponding graphs are as illustrated in Fig. 14.

Now let us denote by $\left(\phi_{c}, v_{c}\left(\phi_{c}\right)\right)$ the exit point (in reverse time) of $W_{c}^{s}\left(P_{c}\right)$ from the region $\{(\phi, v) \mid 0 \leqq \phi \leqq 1, v>0\}$ and define a critical value $c^{*}$, of $c$, as follows:

$$
c^{*}=\inf \left\{c>0 \mid \phi_{c}=1, v_{c}(1)<0\right\} .
$$

Given that for suitable values of $c, W_{c}^{s}\left(P_{c}\right)$ leaves the region $\{(\phi, v) \mid 0 \leqq \phi \leqq 1$, $v<0\}$ on $(0,1)$ this implies that the set in the definition of $c^{*}$ has a lower bound, hence $c^{*}$ is well defined.

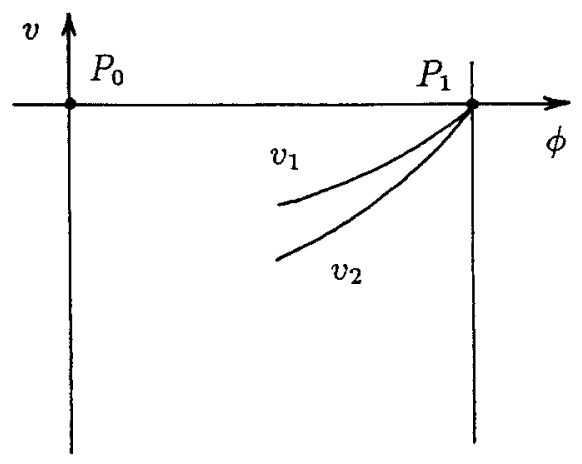

Fig. 14. Geometrical interpretation of the result given in Proposition 7 
We can prove the following lemma:

Lemma 2 (Existence). For $c^{*}$ defined above the trajectory $W_{c^{*}}^{s}\left(P_{c^{*}}\right)$ of $(18)$ connects the saddles $P_{1}$ and $P_{c^{*}}$.

Proof. We use the continuity of the solutions of (18) with respect to $c$ and the monotonicity property given in Proposition 5. The aim is to prove that $\phi_{c^{*}}=1$ and $v_{c^{*}}\left(\phi_{c^{*}}\right)=0$. We will prove it by contradiction. If the above does not occur then we have two possibilities:

1. $\phi_{c^{*}}=1$ and $v_{c^{*}}\left(\phi_{c^{*}}\right)<0$,

2. $0<\phi_{c^{*}}<1$ and $v_{c^{*}}\left(\phi_{c^{*}}\right)=0$.

Consider first possibility 1 . We take values of $c$ less than but close to $c^{*}$. Let us denote by $v_{c}(\phi)$ and $v_{c^{*}}(\phi)$ the solutions of (38) corresponding to the speeds $c$ and $c^{*}$, respectively. Their graphs are the paths of the trajectories $W_{c}^{s}\left(P_{c}\right)$ and $W_{c^{*}}^{s}\left(P_{c^{*}}\right)$. Because of Proposition 5 and continuity, $v_{c}(\phi)>v_{c^{*}}(\phi)$. In particular this holds for $\phi_{c^{*}}=1$. Therefore $0>v_{c}(1)>v_{c^{*}}\left(\phi_{c^{*}}\right)$. But $c^{*}$ is defined as the infimum of the $c$ 's for which $\phi_{c}=1$ and $v_{c}(1)<0$, yet we have exhibited values of $c$ for which $0>v_{c}(1)>v_{c^{*}}\left(\phi_{c^{*}}\right)$. Hence we have obtained a contradiction to the definition of $c^{*}$. Therefore possibility 1 . cannot occur.

We now consider possibility 2 . Here we take values of $c$ larger than but close to $c^{*}$. By using the above notation we have, by Proposition 5 and continuity arguments, that $v_{c}(\phi)<v_{c^{*}}(\phi)<0$. Moreover, $0<\phi_{c^{*}}<\phi_{c}<0$ with $v_{c^{*}}\left(\phi_{c}\right)=v_{c}\left(\phi_{c}\right)=0$. Again we have found values of $c$ which violate the definition of $c^{*}$. Therefore our assumption is false.

\section{Global analysis III: the uniqueness of a sharp type solution and a summary}

In this section we present an alternative uniqueness result of the saddle-saddle heteroclinic trajectory for (18) to that given in [27].

Let $\theta$ be the angle between the positive $\phi$-axis and the vector defined by (18) at $(\phi, v) \in \mathscr{R}_{1}=\{(\phi, v) \mid 0<\phi \leqq 1,-\infty<v<0\}$. For each $(\phi, v) \in \mathscr{R}_{1}$ and for each $c$, we have

$$
\theta(\phi, v ; c)=\tan ^{-1}\left[\frac{-c v-D^{\prime}(\phi) v^{2}-g(\phi)}{D(\phi) v}\right] .
$$

Now we can prove the following proposition:

Proposition 8. For fixed $(\phi, v) \in \mathscr{R}_{1}, \theta(\phi, v ; c)$ is a decreasing function of $c$.

Proof. Let $(\phi, v)$ be an arbitrary fixed point on $\mathscr{R}_{1}$. By using an elementary formula we have

$$
\theta^{\prime}(c)=\frac{-D(\phi) v^{2}}{[D(\phi) v]^{2}+\left[-c v-D^{\prime}(\phi) v^{2}-g(\phi)\right]^{2}}<0 .
$$

The geometrical interpretation of Proposition 8 is shown in Fig. 15. 

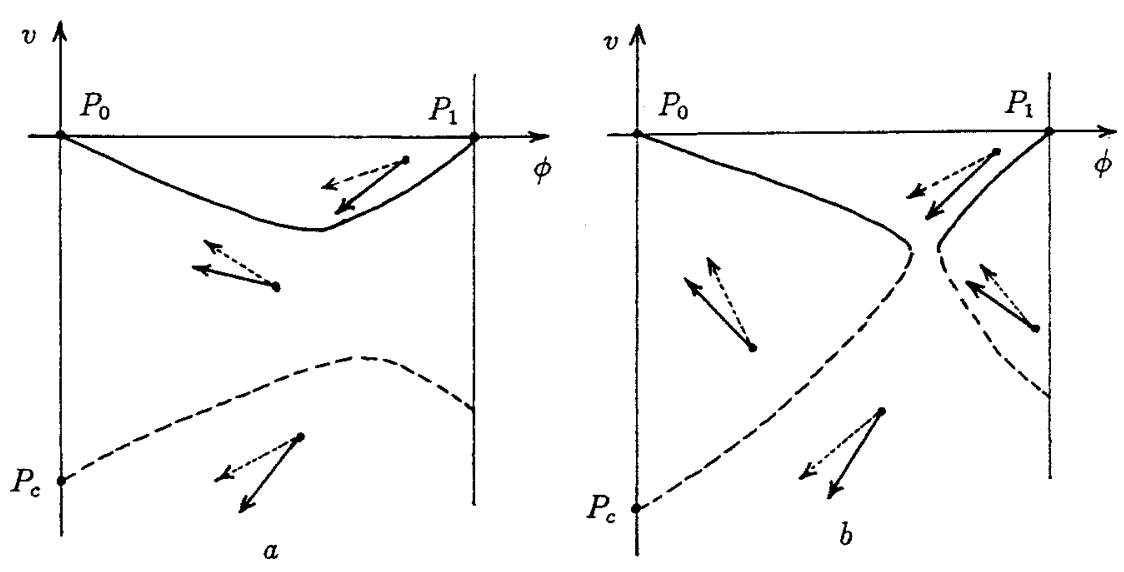

Fig. 15a, b. Geometrical interpretation of Proposition 8 in different regions depending on the vertical null-clines: $\mathbf{a} c^{2}>M$ and $\mathbf{b} c^{2}<M$. The angle $\theta$ is a decreasing function of $c$. The vectors represented by the broken lines correspond to a larger value of $c$ than for those drawn with continuous lines

With Propositions 7 and 8, we can demonstrate the following result on uniqueness of the heteroclinic trajectory for the system (18) connecting $P_{1}$ with $P_{c}$.

Lemma 3 (Uniqueness). For $c^{*}$ defined in the previous section, the trajectory $W_{c^{*}}$ of $(18)$ is the unique one connecting the saddles $P_{1}$ and $P_{c^{*}}$. Moreover, (18) has:

1. No heteroclinic trajectories for $0<c<c^{*}$,

2. A saddle $\left(P_{1}\right)$-saddle-node $\left(P_{0}\right)$ heteroclinic trajectory for $c>c^{*}$.

Proof. Suppose that there are two critical values $c_{1}^{*}$ and $c_{2}^{*}$ of the speed for which the connections $P_{1}-P_{c_{1}^{*}}$ and $P_{1}-P_{c_{2}^{*}}$ exist. Without loss of generality suppose that $c_{1}^{*}<c_{2}^{*}$. Let $v_{1}(\phi)$ and $v_{2}(\phi)$ be the solutions of (38) corresponding to the speeds $c_{1}^{*}$ and $c_{2}^{*}$ respectively, and satisfying the boundary conditions:

$$
v_{1}(1)=v_{2}(1)=0, v_{1}(0)=-\frac{c_{1}^{*}}{D^{\prime}(0)} \quad \text { and } \quad v_{2}(0)=-\frac{c_{2}^{*}}{D^{\prime}(0)} .
$$

By Proposition 7, in a left-neighbourhood of $\phi=1$ the relative positions of $v_{1}$ and $v_{2}$ are as in Fig. 16. This holds for all values of the speed $c>0$, in particular for $c_{1}^{*}$ and $c_{2}^{*}$. The relative positions of $P_{c_{1}^{*}}$ and $P_{c_{2}^{*}}$ on the negative vertical axis is also shown in Fig. 16. This implies the existence of at least one point $\left(\phi_{0}, v_{0}\right) \in \mathscr{R}_{1}$ for which the graphs of $v_{1}$ and $v_{2}$ intersect transversally. This means that the vectors defined by $(18)$ at $\left(\phi_{0}, v_{0}\right)$ for $c_{1}^{*}$ and $c_{2}^{*}$ are as in Fig. 16. This contradicts Proposition 8. Note that both items 1. and 2. are consequences of the continuity of the solutions of (18) with respect to $c$ (see Proposition 3) and of the behaviour for small and large values of $c$. Hence the result. 


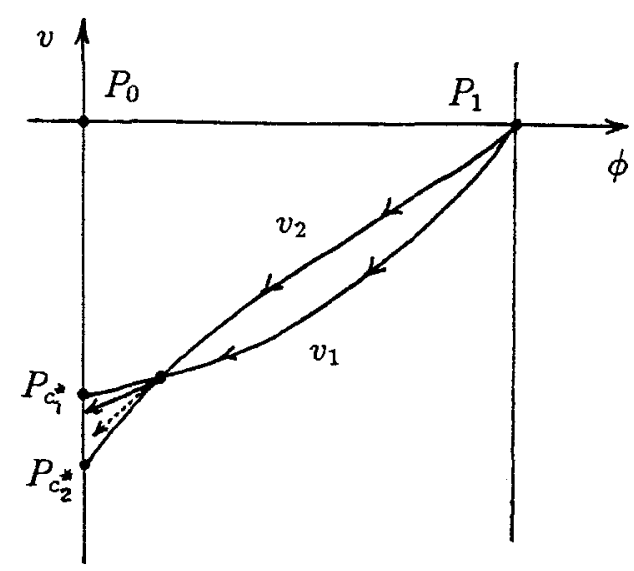

Fig. 16. Proof of Lemma 3. See text for details

For $c^{*}$ as defined in Sect. 6, and using the continuity of the solutions of (18) with respect to $c$, we can summarize all the results in the previous sections in the following theorem which is the main result of this paper:

Theorem 2. If the functions $D$ and $g$ in (10) satisfy the conditions 1 to 4 given above, then the reaction-diffusion equation (10) has:

1. No. t.w.s. for $0<c<c^{*}$,

2. A travelling wave solution $u(x, t)=\phi\left(x-c^{*} t\right)$ of sharp type satisfying: $\phi(-\infty)=1, \phi(\xi)=0 \quad \forall \xi \geqq \xi^{*} ; \quad \phi^{\prime}(-\infty)=0, \quad \phi^{\prime}\left(\xi^{*}\right)=-\frac{c^{*}}{D^{\prime}(0)} \quad$ and $\phi^{\prime}(\xi)=0 \forall \xi \geqq \xi^{*}$,

3. A monotone decreasing travelling wave solution of front type satisfying $\phi(-\infty)=1$ and $\phi(+\infty)=0$ for each $c>c^{*}$.

\section{Illustrative examples}

Here we apply the results of the above sections to different non-linear degenerate parabolic equations corresponding to several forms of the diffusion term $D$ and of the kinetic part $g$ in (10). The numerical simulations that we will carry out are on the phase portrait and on the solutions of the full PDE. In this section we use a program written by Malajovich [20] which draws the phase portrait of two-dimensional autonomous systems of ODEs. This program uses a Runge-Kutta method to solve the ODE system. We obtain the solution of the full PDE by using the NAG library routine DO3PGF, which replaces the space derivative by finite differences to approximate the PDE by a system of ODEs. This system is integrated forward in time using Gear's method. We also plot the speed of the t.w.s. as time increases.

Example 1. Consider the degenerate parabolic equation

$$
\frac{\partial u}{\partial t}=\frac{\partial}{\partial x}\left[\left(\beta u+u^{2}\right) \frac{\partial u}{\partial x}\right]+u(1-u),
$$

where $\beta>0$. 
Clearly the functions $D(u)=\left(\beta u+u^{2}\right)$ and $g(u)=u(1-u)$ satisfy the conditions imposed in the previous sections and therefore all the above results hold.

Suppose that (41) has a travelling wave solution $u(x, t)=\phi(x-c t) \equiv \phi(\xi)$. Setting $v=\phi^{\prime}(\xi)$ we have that the second order ODE for $\phi$ can be written as the following singular system

$$
\begin{aligned}
\phi^{\prime} & =v \\
v^{\prime} & =\frac{1}{\left(\beta \phi+\phi^{2}\right)}\left[-c v-(\beta+2 \phi) v^{2}-\phi(1-\phi)\right] .
\end{aligned}
$$

Introducing the parameter $\tau$ as in (15) with $D(\phi)=\beta \phi+\phi^{2}$, we get a new system without the singularity

$$
\begin{aligned}
& \dot{\phi}=\left(\beta \phi+\phi^{2}\right) v \\
& \dot{v}=-c v-(\beta+2 \phi) v^{2}-\phi(1-\phi)
\end{aligned}
$$

whose equilibrium points are $P_{0}=(0,0), P_{1}=(1,0)$ and $P_{c}=\left(0, v^{*}\right)$, where $v^{*}=-\frac{c}{\beta}$.

In Fig. 17 we show the numerically calculated phase portraits of the system (43) for $\beta=2$ and for different values of $c$. These agree with the analysis of the previous sections. In particular, this figure shows that system (43) has a saddle-saddle heteroclinic trajectory for values of $c$ close to 1.0979 which, by Lemma 3, must be unique (see Fig. 17b). Corresponding to this connecting trajectory we have a travelling wave solution of sharp type for equation (41). This is because $\phi(-\infty)=1$ and as $\tau$ increases $\phi$ tends to 0 but $\dot{\phi}$ tends to $-1.0979 / 2$.

Figure 17a shows the non-existence of heteroclinic trajectories, which means non-existence of t.w.s. for (41). Figure 17c shows a saddle to saddlenode connection which was also predicted by the analysis of the previous sections. In this case, since $\phi(-\infty)=1$ and $\phi(+\infty)=\dot{\phi}(+\infty)=0$ with $\dot{\phi}(\tau)<0 \forall \tau \in(-\infty,+\infty)$, corresponding to the above connection we have a monotonic decreasing front type solution for (41).

In Fig. 18a we show the numerical solution of the PDE (41) for the step function initial condition

$$
u(x, 0)=u_{0}(x)= \begin{cases}1, & \forall x \in\left(-x_{\max }, 0\right) \\ 0, & \forall x \in\left(0, x_{\max }\right)\end{cases}
$$

where $x_{\max }>0$ and such that $2 x_{\max }$ is the size of the space interval on which the equation is solved. For numerical purposes it is enough to consider sufficiently large values of $x_{\max }$. The boundary conditions are

$$
u\left(-x_{\max }, t\right)=1 \quad \text { and } u\left(x_{\max }, t\right)=0 \forall t \in\left[0, t_{\max }\right]
$$

where $\left[0, t_{\max }\right]$ is the time interval on which the system is solved.

Each graph in this figure corresponds to the solution of the PDE at regular time intervals of duration $t_{\max } / n$, where $n$ in the number of iterations. Clearly this wave is of sharp type. In Fig. $18 \mathrm{~b}$ we show the speed of a selected point 

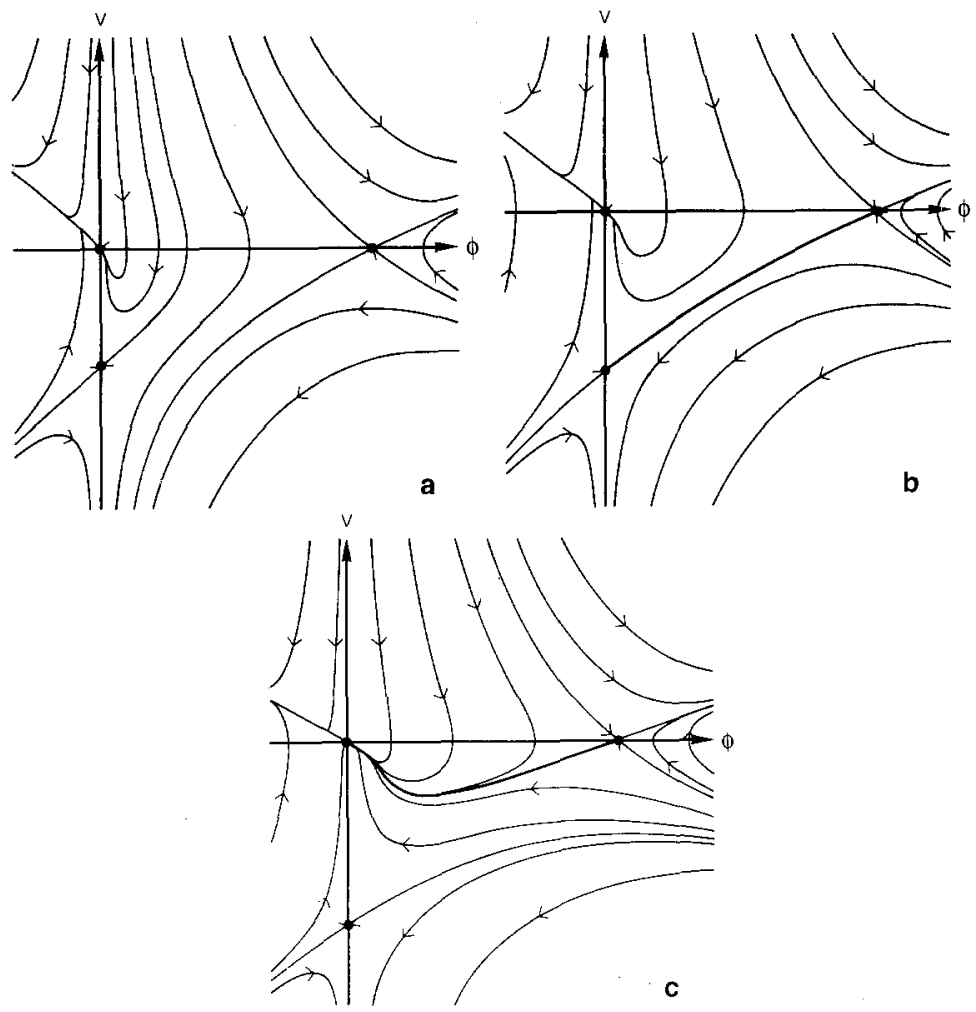

Fig. 17a-c. Phase portraits of the system (43) for different values of $c$ : a $0<c<1.0979$; here we take $c=0.81$; there are no heteroclinic trajectories. $\mathbf{b} c=1.0979$; the figure shows a good approximation to the saddle-saddle connection. $c c>1.1$; here we take $c=1.5$. The figure illustrates a saddle-saddle-node trajectory
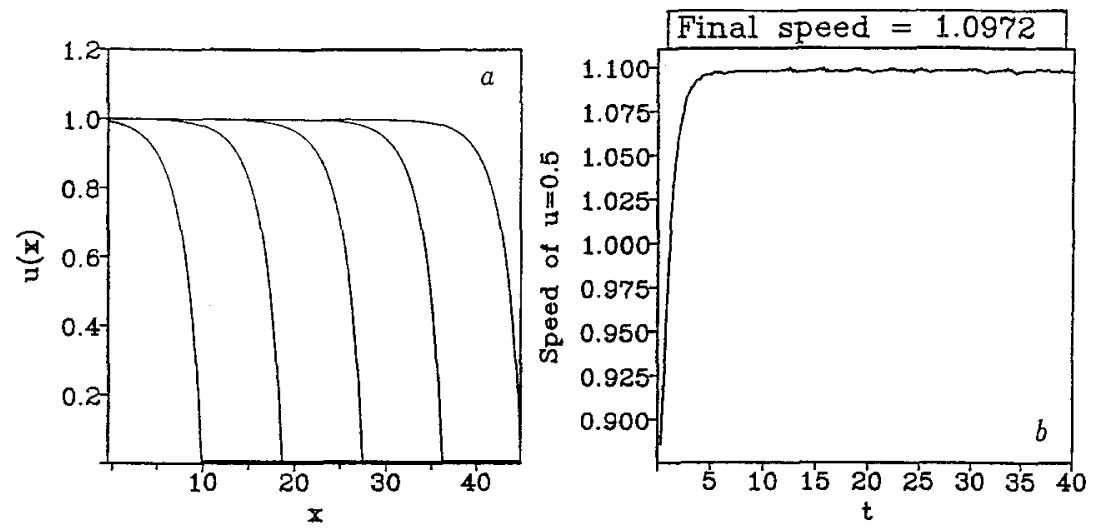

Fig. 18a, b. Numerical solution of (41): a The sharp type solution at regular time intervals. b Diagram of the speed as a function of time. The calculated final speed $c^{*} \approx 1.089$ agrees well with that in Fig. 17b 
$(u=0.5)$ on the solution profile. Note that the final speed agrees closely with that calculated for the saddle-saddle heteroclinic connection.

Example 2. Here we consider the equation

$$
\frac{\partial u}{\partial t}=\frac{\partial}{\partial x}\left[\left(1-e^{-u}\right) \frac{\partial u}{\partial x}\right]+u(1-u)
$$

We follow the same procedure as before. After substituting $u(x, t)=\phi(x-c t)$ into (44), writing down the singular system of ODEs and introducing the parameter $\tau$ as in (15) with $D(\phi)=(1-\exp (-\phi))$, we get the nonsingular system

$$
\begin{aligned}
\dot{\phi} & =\left(1-e^{-\phi}\right) v \\
\dot{v} & =-c v-e^{-\phi} v^{2}-\phi(1-\phi),
\end{aligned}
$$

with equilibrium points: $P_{0}=(0,0), P_{1}=(0,1)$ and $P_{c}=(0,-c)$. The local and global behaviour of (45) is as in previous sections. Figure 19 shows the phase
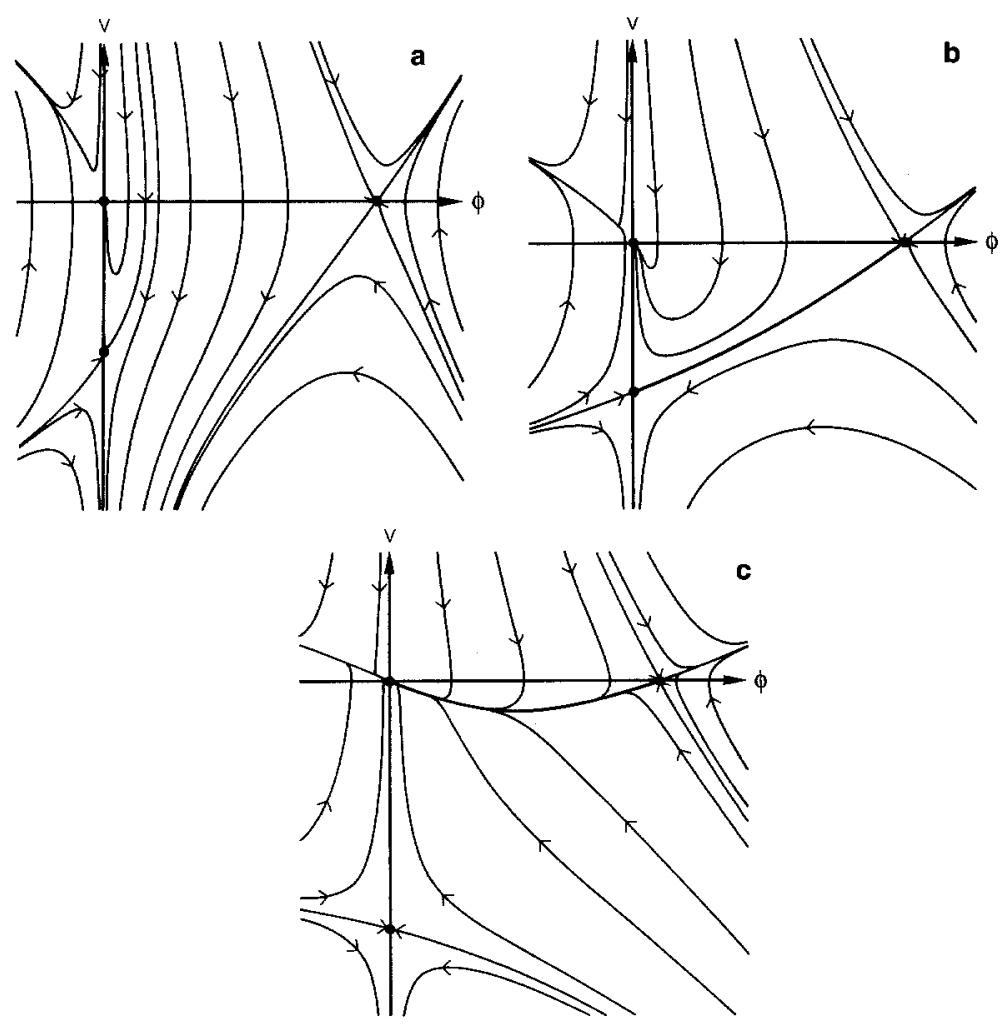

Fig. 19a-c. Phase portrait of (45) for different values of $c$ : a $c=0.4$; there are no heteroclinic connections. b $c=0.645$; this is a good approximation to the critical value $c^{*}$ for which there exists the heteroclinic saddle-saddle trajectory. $\mathbf{c} c=1.5$; here we have a saddle-saddle-node connection 

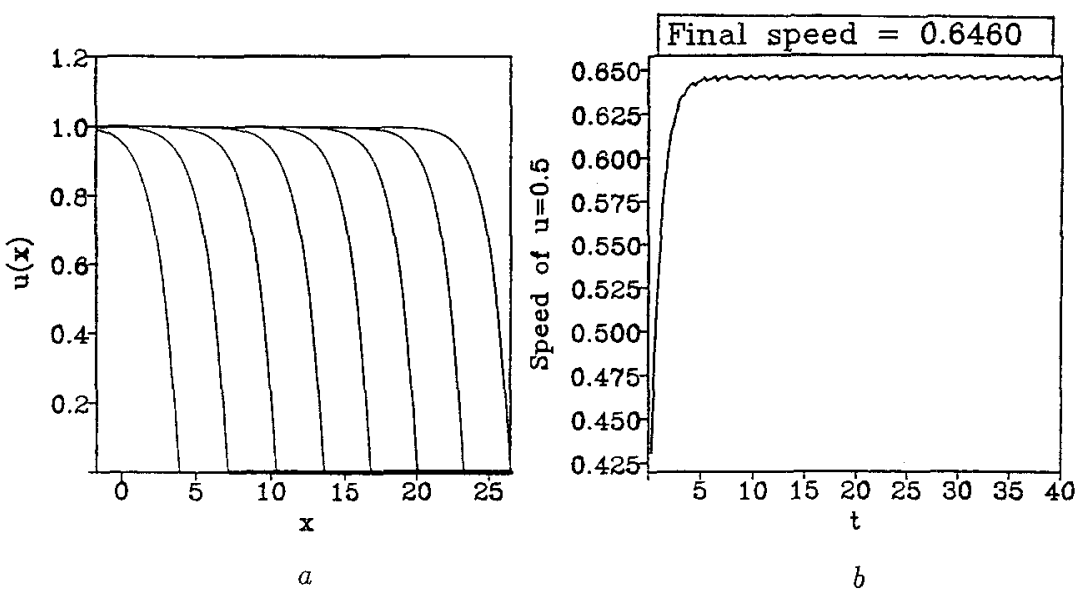

Fig. 20a, b. Numerical simulation for (44). a Sharp front solutions at regular time intervals and $\mathbf{b}$ diagram of the speed of the sharp solution as a function of time

portrait of (45) for different values of $c$. These numerical simulations strongly suggest that the critical value $c^{*}$ of the speed $c$ for which there exists a heteroclinic saddle-saddle connection is $\approx 0.645$ (Fig. 19b). The behaviour for $0<c<0.645$ is shown in Fig. 19a and that for $c>0.645$ is shown in Fig. 19c.

If we solve numerically (44) for the step function initial condition as in Example 1, we obtain the sharp type solution profile shown in Fig. 20a. Figure $20 \mathrm{~b}$ shows the dependence of the speed $c$ on the time. After a transient phase, the speed reaches a steady value which agrees with the results from the phase plane simulations.

Acknowledgement. PKM would like to thank the Department of Applied Mathematics, University of Washington, Seattle for its hospitality, and for support from the Robert F. Philip Endowment. We thank Dr J.A. Sherratt for helpful discussions.

\section{References}

1. Andronov, A. A., Leontovich, E. A., Gordon, I.I., Maier, A. G.: Theory of dynamical systems on a plane. Israel Program for Scientific Translations, Jerusalem 1972

2. Arnold, V. I.: Ordinary differential equations. Cambridge: MIT Press 1980

3. Aronson, D. G.: Density-dependent interaction-systems. In: Steward, W. H., Ray, W. H., Conley, C. C. (eds.) Dynamics and modelling of reactive systems. New York: Academic Press 1980

4. Aronson, D. G.: The role of the diffusion in mathematical population biology: Skellam revisited. In: Fasano, A., Primicerio, M. (eds.) Lecture Notes in Biomathematics 57. Berlin Heidelberg New York: Springer 1985

5. Arrowsmith, D. K., Place, C. M.: An introduction to dynamical system. Cambridge: Cambridge University Press 1990

6. Britton, N. F.: Reaction-diffusion equations and their applications to biology. New York: Academic Press 1986 
7. Carl, E. A.: Population control in Arctic ground squirrels. Ecology 52, 395-413 (1971)

8. Carr, J.: Applications of centre manifold theory. Berlin Heidelberg New York: Springer 1981

9. de Pablo, A., Vázquez, J. L.: Travelling waves in finite propagation in a reactiondiffusion equation. J. Diff. Equ. 93, 19-61 (1991)

10. Engler, H.: Relations between travelling wave solutions of quasilinear parabolic equations. Proc. Am. Math. Soc. 93, 297-302 (1985)

11. Fife, P. C.: Mathematical aspects of reacting and diffusing systems. Berlin Heidelberg New York: Springer 1979

12. Fisher, R. A.: The wave of advance of advantageous genes. Ann. Eugen 7, 353-369 (1937)

13. Grindrod, P., Sleeman B. D.: Weak travelling wave fronts for population models with density-dependent dispersion. Math. Meth. Appl. Sci. 9, 576-586 (1987)

14. Gurney, W. S. C., Nisbet, R. M.: The regulation of inhomogeneous population. J. Theor. Biol. 441-457 (1975)

15. Gurney, W. S. C., Nisbet, R. M.: A note on non-linear population transport. J. Theor. Biol. 56, 249-251 (1976)

16. Gurtin, M. E., MacCamy, R. C.: On the diffusion of biological populations. Math. Biosci. 33, 35-49 (1977)

17. Hadeler, K. P.: Travelling fronts and free boundary value problems. In: Albretch, J., Collatz, L., Hoffman, K. H. (eds.) Numerical Treatment of Free Boundary Value Problems. Basel: Birkhauser 1981

18. Hadeler, K. P.: Free boundary problems in biological models. In: Fasano, A., Primicerio, M. (eds.) Free Boundary Problems: Theory and Applications, Vol. II. London: Pitman 1983

19. Kolmogorov, A., Petrovsky, I., Piskounov, I. N.: Study of the diffusion equation with growth of the quantity of matter and its applications to a biological problem. (English translation containing the relevant results) In: Oliveira-Pinto, F., Conolly, B. W. (eds.) Applicable mathematics of non-physical phenomena. New York: Wiley 1982

20. Malajovich, G.: Programa Tracador de Diagramas de Fase. Instituto de Matematica. Universidade Federal do Rio de Janeiro, Brazil 1988

21. Montroll, E. W., West, B. J.: On an enriched collection of stochastic processes. In: Montroll, E. W., Lebowitz, J. L. (eds.) Fluctuation phenomena. Amsterdam: NorthHolland 1979

22. Murray, J. D.: Mathematical biology. Biomathematics Texts 19. Berlin Heidelberg New York: Springer 1989

23. Myers, J. H., Krebs, Ch. J.: Population cycles in rodents. Sci. Am. 6, 38-46 (1974)

24. Newman, W. I.: Some exact solutions to a non-linear diffusion problem in population genetics and combustion. J. Theor. Biol. 85, 325-334 (1980)

25. Newman, W. I.: The long-time behaviour of the solution to a non-linear diffusion problem in population genetics and combustion. J. Theor. Biol, 104, 473-484 (1983)

26. Sánchez-Garduño, F.: Travelling waves in one-dimensional degenerate non-linear reaction-diffusion equations. D. Phil. thesis., University of Oxford (1993)

27. Sánchez-Garduño, F., Maini, P. K.: Travelling wave phenomena in some degenerate reaction-diffusion equations. J. Diff. Equ. (in press)

28. Sánchez-Garduño, F., Maini, P. K.: Travelling wave phenomena in non-linear diffusion degenerate Nagumo equations (submitted)

29. Shiguesada, N., Kawasaki, K., Teramoto, E.: Spatial segregation of interacting species. J. Math. Biol. 79, 83-99 (1979)

30. Skellam, J. G.: Random dispersal in theoretical populations. Biometrika 38, 196-218 (1951)

31. Skellam, J. G.: The formulation and interpretation of mathematical models of diffusionary processes in population biology. In: Bartlett, M. S. et al. (eds.) The mathematical theory of the dynamics of biological population. New York: Academic Press 1973 